\title{
Ion Cyclotron Resonance Heating for tungsten control in various JET H-mode scenarios
}

\author{
M.Goniche ${ }^{1}$, R.J.Dumont ${ }^{1}$, V.Bobkov ${ }^{2}$, P.Buratti ${ }^{3}$, S.Brezinsek ${ }^{4}$, C.Challis ${ }^{5}$, L.Colas ${ }^{1}$,

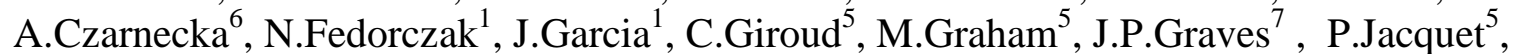 \\ E.Lerche $^{8}$, P.Mantica ${ }^{9}$, I.Monakhov ${ }^{5}$, P.Monier-Garbet ${ }^{1}$, M.F.F. Nave ${ }^{10}$, C.Noble ${ }^{5}$, I.Nunes ${ }^{10}$, \\ T.Pütterich ${ }^{2}$, F.Rimini ${ }^{5}$, M.Valisa ${ }^{11}$, D. Van Eester ${ }^{8}$ and JET Contributors* \\ ${ }^{I}$ CEA, IRFM, F-13108 Saint-Paul-lez-Durance, France. \\ ${ }^{2}$ Max-Planck-Institut für Plasmaphysik, EURATOM-Assoziation, Garching, Germany \\ ${ }^{3}$ ENEA, C.R. Frascati, Via E. Fermi 4500044 Frascati (RM), Italy \\ ${ }^{4}$ Forschungszentrum Jülich, EURATOM Association, Jülich, Germany \\ ${ }^{5}$ Euratom/CCFE Fusion Association, Culham Science Centre, Abingdon, OX14 3DB, UK \\ ${ }^{6}$ Association Euratom-IPPLM, Hery 23, 01-497 Warsaw, Poland \\ ${ }^{7}$ Ecole Polytechnique Fédérale de Lausanne (EPFL), Centre de Recherches en Physique des \\ Plasmas, 1015 Lausanne, Switzerland \\ ${ }^{8}$ Association EURATOM-Belgian State, LPP-ERM-KMS, TEC partner, Brussels, Belgium \\ ${ }^{9}$ Istituto di Fisica del Plasma 'P.Caldirola', Consiglio Nazionale delle Ricerche, Milano, Italy \\ ${ }^{10}$ Instituto de Plasmas e Fusão Nuclear, IST, Universidade de Lisboa, Portugal \\ ${ }^{11}$ Consorzio RFX, Consiglio Nazionale delle Ricerche, Padova, Italy \\ * See the Appendix of F. Romanelli et al., Proc. of the 25th IAEA Fusion Energy \\ Conference, 2014, St. Petersburg, Russia
}

\begin{abstract}
Ion Cyclotron Resonance Heating (ICRH) in the minority scheme provides central ion heating and acts favorably on the core tungsten transport. Full wave modeling shows that after collisional redistribution, the ratio of power transferred to the ions and the ielectrons vary little with the minority (hydrogen) concentration $n_{H} / n_{e}$ but the high-Z impurity screening provided by the fast ions temperature increases with

The power radiated by tungsten in the core of the JET discharges has been analyzed on a large database covering the 2013-2014 campaign. In the baseline scenario with moderate plasma current $(I p=2.5 M A)$ ICRH provides efficient effect on tungsten transport and when the ICRH power is increased, the tungsten radiation peaking evolves as predicted by the neo-classical theory. At higher current (3-4MA), tungsten accumulation can be avoided with 5MW of ICRH power with high gas injection rate only. For discharges in the hybrid scenario, the strong initial peaking of the density leads to strong tungsten accumulation.
\end{abstract} When this initial density peaking is slightly reduced, with an ICRH power in excess of 
$4 M W$,very low tungsten concentration in the core ( 10-5) is maintain for 3 seconds. MHD activity plays a key role in tungsten transport and modulation of the tungsten radiation during a sawtooth cycle is correlated to the fishbone activity triggered by the fast ion pressure gradient.

\section{Introduction}

In order to prepare the ITER plasma scenarios, the divertor of the JET tokamak has been changed in 2011and is now made of either plain tungsten tiles or tungsten-coated tiles (ITER-like wall, nicknamed ILA). Tungsten like all high $\mathrm{Z}$ atoms, has a very high radiation capability and therefore detrimental effect on plasma performance if this impurity concentration in the core is too high. It is generally admitted that the concentration has to be kept below $10^{-4}$. Neoclassical theory predicts an inward pinch leading to an accumulation of high $\mathrm{Z}$ impurities in the very core of the plasma $(\mathrm{r} / \mathrm{a}<0.2)$. Moreover, strong plasma rotation occurs in neutral beam heated plasmas and the resulting centrifugal force is the cause of poloidal asymmetry enhancing the radiation on the low field side of the plasma.

This deleterious effect is mitigated by temperature screening and the sign of convection can be inversed when the ion temperature gradient is sufficiently large with respect of the ion density gradient. Under some assumptions which can be found in ref. (Angioni 2014, Casson 2014), when the poloidal asymmetries are not considered the tungsten flux can be expressed as

$\Gamma \mathrm{W} \sim \mathrm{n}_{\mathrm{i}} \mathrm{T}_{\mathrm{i}} v_{\mathrm{iW}} \mathrm{Z}_{\mathrm{W}}\left(\mathrm{R} / \mathrm{L}_{\mathrm{ni}}-0.5 \mathrm{R} / \mathrm{L}_{\mathrm{Ti}}\right) \sim \mathrm{n}_{\mathrm{i}} \mathrm{T}_{\mathrm{i}}^{-1 / 2} \mathrm{Z}_{\mathrm{W}}\left(\mathrm{R} / \mathrm{L}_{\mathrm{ni}}-0.5 \mathrm{R} / \mathrm{L}_{\mathrm{Ti}}\right)$

where $\mathrm{L}_{\mathrm{X}}$ is the inverse of the logarithmic gradient $\mathrm{d}[\log (\mathrm{X})] / \mathrm{dR}$. However these poloidal asymmetries, arising from the centrifugal force, are important for heavy impurities and an additional term with a positive sign (inward flux) has to be added to the expression of the tungsten flux.

In addition turbulent transport can contribute to impurity transport, although for heavy impurities such as tungsten, modelling combining neoclassical and gyrokinetic codes indicates that neoclassical transport dominates on turbulent transport [Angioni 14].

Ion cyclotron resonance heating (ICRH) can beneficially affect the tungsten transport for several reasons. Firstly, depending on the details of the minority heating scheme (mainly the minority species and concentration) and the plasma parameters (mainly the electron and ion temperatures), a large part of wave energy can be transferred to the bulk ions either by 
Coulomb collisions with the fast minority ions or by direct damping on the majority ions [Mantsinen 99, Dumont 13, Mantsinen 15]. Secondly, the fast ion tail can contribute to the impurity screening [Casson 2014] as the screening scales as $\mathrm{n}_{\mathrm{f}} \mathrm{T}_{\mathrm{f}}^{-1 / 2}\left(\mathrm{R} / \mathrm{L}_{\mathrm{Tf}}\right)$ and very local deposition of ICRH leads to normalized gradient $\mathrm{R} / \mathrm{L}_{\mathrm{Tf}}$ which can exceed 50 . Thirdly, the poloidal asymmetry of tungsten radiation, due to the centrifugal force in strongly rotating plasmas with NBI heating, is reduced by the temperature anisotropy of the minority species [Bilato 14]. In addition to the neo-classical effects, turbulent transport can be enhanced by core electron heating provided by ICRH waves. This effect is very likely a major player in Asdex Upgrade low-collisionality plasmas where a moderate ECRH power on top of the NBI power allows a control of the core tungsten concentration [Püterrich 13?]. Finally, MHD activity and particularly the $(1,1)$ mode, can allow a flushing of the tungsten from the core of the discharge.

The drawback of using ICRH in metallic environment is related to enhanced high $\mathrm{Z}$ material sputtering arising from ions accelerated by the DC rectified potential in the near field of the antenna [Jacquet 14, Bobkov 14, Czarnecka 14]. This potential can exceed 100V and energy of light impurities, such as beryllium for the ILW case, is above the sputtering threshold of tungsten. Therefore the main issue when using ICRH is whether or not the beneficial effect on particle transport overcomes the detrimental increase of the tungsten source.

Effect of ICRH on transport of metallic impurities has been studied on JET with the carbon wall by an injection of nickel and molybdenum performed by a laser blow-off technique [Valisa 2011]. For L-mode discharges performed at rather low plasma current $\left(\mathrm{I}_{\mathrm{p}}=1.5-2.3 \mathrm{MA}\right)$, it was found that the convection was reversed from inward to outward when the ICRH power exceeded $3 \mathrm{MW}$ in the $\mathrm{H}_{\text {or }} \mathrm{He}^{3}$ minority heating scheme. With the ILW, beneficial effect of ICRH power for [Goniche 14, Casson14, Giroud15, Lerche 14]. In the hybrid scenario, central ICRH heating mitigates the tungsten accumulation at least in the early phase of the high power phase [Mantica 15].

In this paper, tungsten radiation from ICRH-heated plasmas is analyzed on a large database covering the JET baseline scenario with plasma current in the 2.5-4MA range $(\mathrm{Bt}=2.7-3.8 \mathrm{~T})$ and the hybrid scenario at 2.5MA $(\mathrm{Bt} \sim 2.9 \mathrm{~T})$. The ICRH power could be extended to $6 \mathrm{MW}$ with the ILW, thanks to the beneficial effect of gas injection from the torus valve located either in the mid-plane or at the top of the vacuum vessel [Lerche 14b, 
Jacquet 14]. However The RF power does not exceed $20 \%$ of the total injected power, mainly provided by the neutral beams.

Radiation and concentration of tungsten in the core of the discharge are derived from the soft X-ray (SXR) diagnostic following the inversion procedure detailed elsewhere [Pütterich 12], providing a poloidal map of these quantities as long as the electron temperature exceeds $1.5 \mathrm{keV}$ which is the case in the analyzed discharge at least in the inner half of the plasma $(\mathrm{r} / \mathrm{a} \leq 0.5)$ and in most cases in even a wider volume. For the calculation of the radiated power and tungsten concentration, it is assumed that all the radiation is caused by tungsten when other high-Z metals (nickel and molybdenum for these experiments) could also contribute. From the poloidal asymmetry of the SXR radiation, the toroidal rotation was calculated and compared to that obtained from the charge exchange spectroscopy diagnostic. It was found a very good agreement for discharges performed from the beginning to the end of the 2014 campaign and it can be concluded that the SXR radiation from lighter metal impurities ( $\mathrm{Mo}$ and $\mathrm{Ni}$ ) does not contribute significantly in the plasma core $(\mathrm{r} / \mathrm{a}<0.5)$. As a rule of thumb a power volume density of $0.1 \mathrm{MW} / \mathrm{m}^{3}$ corresponds to a tungsten concentration of $10^{-4}$ which is usually considered as the upper limit to maintain high performance. The tungsten peaking factor is defined as the ratio of the flux surface-averaged radiation power densities $\mathrm{PF}_{0.3}=\mathrm{P}_{\mathrm{rad}}-\mathrm{W}(\mathrm{r} / \mathrm{a}=0) / \mathrm{P}_{\mathrm{rad}}-\mathrm{W}(\mathrm{r} / \mathrm{a}=0.3)$

\section{Modelling of the ICRH damping and temperature screening by the fast ions}

In order to investigate how the ICRH power is damped when the hydrogen minority concentration $n_{H} / n_{e}$ is varied, the EVE/AQL code [Dumont 2009, Dumont 2013] was run for a high power discharge $\left(\mathrm{P}_{\mathrm{NBI}}=24 \mathrm{MW}, \mathrm{P}_{\mathrm{ICRH}}=4 \mathrm{MW}\right)$ with $\mathrm{n}_{\mathrm{H}} / \mathrm{n}_{\mathrm{e}}$ varying between $0.25 \%$ and $15 \%$ while keeping the electron and bulk ion temperature profile identical $\left(\mathrm{T}_{\mathrm{e}}(0)=7.5 \mathrm{keV}\right.$ and $\left.\mathrm{T}_{\mathrm{i}}(0)=7.5 \mathrm{keV}\right)$. When the minority concentration is increased, wave energy is transferred from the deuterium (majority $2^{\text {nd }}$ harmonic heating scheme) to the hydrogen (minority $1^{\text {st }}$ harmonic heating scheme) whereas the fraction damped directly on the electrons does not vary much (Figure 1-a). The fast ion distribution is centered on a normalized radius $\mathrm{r} / \mathrm{a} \sim 0.15$, corresponding in that case to the position of the ion cyclotron resonance layer, with an effective temperature $\left(\mathrm{T}_{\text {eff }}=\left(\mathrm{T}_{/ /}+2 \mathrm{~T}_{\text {perp }}\right) / 3\right)$ strongly decreasing with increasing $\mathrm{n}_{\mathrm{H}} / \mathrm{n}_{\mathrm{e}}$ (figure 1-b). The fast ion tail does not extend beyond the normalized radius $\mathrm{r} / \mathrm{a}=0.35$ and the hydrogen temperature out of this limit is the temperature of the background 
ions. The temperature anisotropy $\mathrm{T}_{\text {perp }} / \mathrm{T}_{/ /}$also decreases from 4.5 at low concentration $\left(\mathrm{n}_{\mathrm{H}} / \mathrm{n}_{\mathrm{e}}=1 \%\right)$ to 1.2 at high concentration $\left(\mathrm{n}_{\mathrm{H}} / \mathrm{n}_{\mathrm{e}}=15 \%\right)$ (Figure $\left.2-\mathrm{a}\right)$.
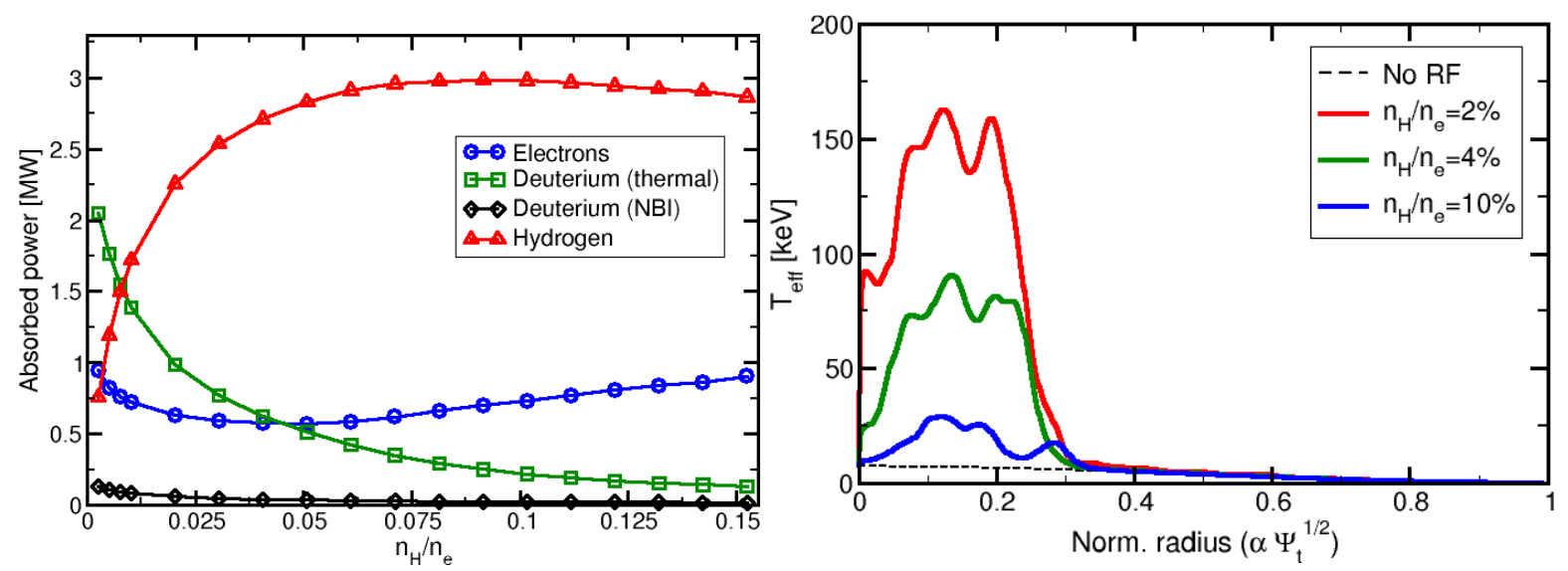

Figure 1 a) Wave power damping on the different species b) Radial profile of the effective temperature $\left(T_{\text {eff }}=\left(T_{/ /}+2 T_{\text {perp }}\right) / 3\right) . \quad P_{N B I}=24 M W, \quad P_{I C R H}=4 M W, \quad n_{e}(0)=6.5 \times 10^{19} \mathrm{~m}^{-3}, \quad T_{e}(0)=7.5 \mathrm{keV}$, $T_{i}(0)=7.5 \mathrm{keV}$.

After collisional redistribution, the fraction of power transferred to the D ions increases when $\mathrm{n}_{\mathrm{H}} / \mathrm{n}_{\mathrm{e}}$ increases from $4 \%$ to $12 \%$ and then levels off for larger concentration (Figure 2b). This is the result of the thermal contribution to the hydrogen ions energy as the minority concentration increases. However the ratio of the power transferred to the electrons and ions varies in a limited range (44-56\%). For high hydrogen concentration, power damping decreases and consequently reduces the effective ion heating efficiency. Experiments carried out with hydrogen minority concentration varying between $2 \%$ and $22 \%$ did not show evidence on significant increase of the ion temperature at high concentration (Van Eester 14, Lerche14).
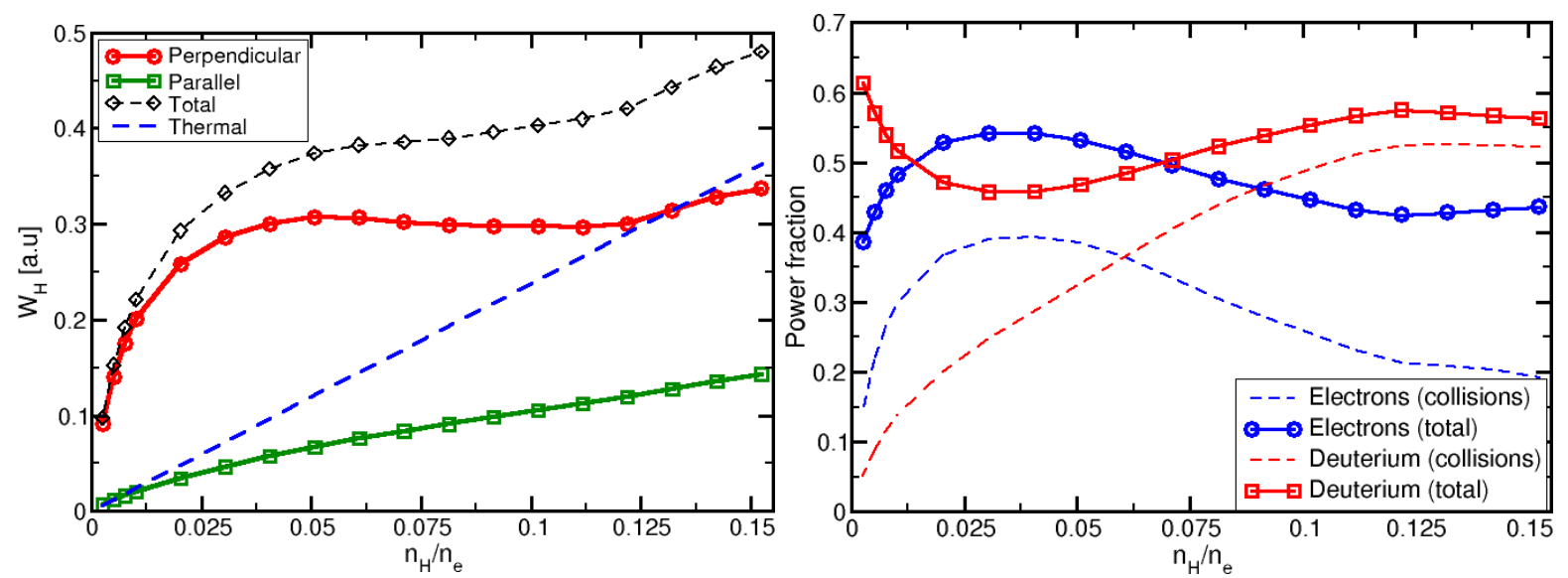
Figure 2 a) Perpendicular and parallel energy of the hydrogen ions b) Fraction of RF power transferred to the electrons and ions after collisional redistribution (solid lines) and fraction of the power transferred from the hydrogen to the as a function of the minority concentration $n_{H} / n_{e}$. $P_{N B I}=24 M W, P_{I C R H}=4 M W, n_{e}(0)=6.5 \times 10^{19} \mathrm{~m}^{-3}, T_{e}(0)=7.5 \mathrm{keV}, T_{i}(0)=7.5 \mathrm{keV}$.

From the modelled fast ion temperature profile, the temperature screening factor $\mathrm{n}_{\mathrm{f}} \mathrm{T}_{\mathrm{f}}^{-}$ ${ }^{1 / 2}\left(\mathrm{R} / \mathrm{L}_{\mathrm{Tf}}\right)$ was computed and compared to the temperature screening factor provided by the thermal deuterium ions. This screening factor is found to be the highest at $\mathrm{r} / \mathrm{a} \sim 0.28$ for the low range of hydrogen concentration $\left(3 \%<\mathrm{n}_{\mathrm{H}} / \mathrm{n}_{\mathrm{e}}<10 \%\right)$ and the maximum moves inwards for higher concentrations (figure 3). For the usual cases of ICRH with $n_{H} /$ ne $<10 \%$, the screening provided by the fast $\mathrm{H}$ ions is much lower (at least a factor 3 ) than that provided by the thermal $\mathrm{D}$ ions. The screening by fast ions and thermal ions, with $\mathrm{n}_{\mathrm{H}} / \mathrm{ne}=9 \%$, was found of the same order in a previous work [Casson 2015]. Taking into account, the large uncertainty on the temperature gradient for the calculation of the screening factor, the screening factor has an error bar of at least $\pm 30 \%$ but surely this factor increases with $n_{H} /$ ne although the temperature of the fast ions sharply decreases.

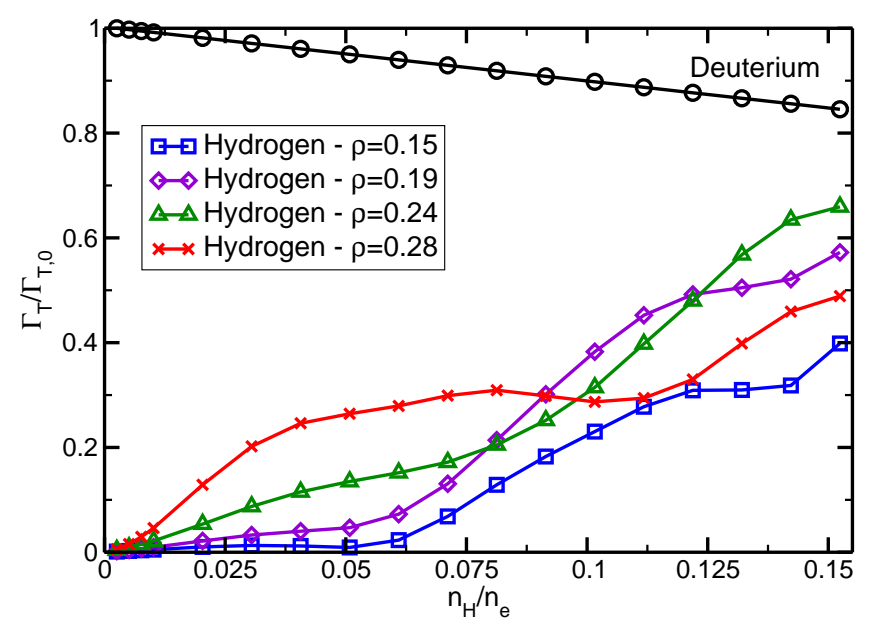

rigure S. І emperature screening Jactor $\Lambda_{T}$ normalizea to the case of a pure D plasma $\Gamma_{T, 0}$ for fast $H$ and thermal $D$ ions.

\section{Effect of the ion cyclotron resonance position and MHD activity.}


In order to optimize the heating scenario, the ion cyclotron resonance position $\left(\mathrm{R}_{\mathrm{IC}}\right)$ was varied either by ramping the toroidal field at constant current ( $\mathrm{Ip}=2.5 \mathrm{MA})$ [Lerche 14] or by ramping both the toroidal field and the plasma current at constant safety factor $\left(\mathrm{q}_{95}=3.5\right)$ [Graves 15]. The ICRH power is in the 3.2-3.8MW range when the total injected power (NBI+ICRH) lies between 19MW and 22MW. Hydrogen concentration is relatively high for the Ip-constant discharges $(\mathrm{X}[\mathrm{H}]=7-9 \%)$ and low for the q-constant discharges $(\mathrm{X}[\mathrm{H}]=3-5 \%)$.

Central electron temperature, measured before the sawtooth crash, increases from $\sim 4 \mathrm{keV}$ to $\sim 5.5 \mathrm{keV}$ when the IC resonance layer is moved from the high field side $\left(\mathrm{R}_{\mathrm{IC}^{-}}\right.$ $\left.\mathrm{R}_{\mathrm{mag}}=-0.15 \mathrm{~m}\right)$ to the low field side $\left(\mathrm{R}_{\mathrm{IC}}-\mathrm{R}_{\mathrm{mag}}=0.20 \mathrm{~m}\right)$. For the highest temperature $\left(\mathrm{T}_{\mathrm{e}}>5 \mathrm{keV}\right.$ ), a 1.3s long sawtooth-free period is obtained (Fig.4-a). In the case of the qconstant discharges, the IC resonance position is ramped on the low-field side from $\mathrm{R}_{\mathrm{IC}^{-}}$ $\mathrm{R}_{\mathrm{mag}}=0.35 \mathrm{~m}$ to $\mathrm{R}_{\mathrm{IC}}-\mathrm{R}_{\mathrm{mag}}=0.10 \mathrm{~m}$, except the pulse 85084 which have stationary plasma parameters. Central temperature is found to be maximum for $\mathrm{R}_{\mathrm{IC}}-\mathrm{R}_{\mathrm{mag}}=0.05-0.2 \mathrm{~m}$, depending on the scenario. For the same discharges, the normalized logarithmic gradient of the temperature $\mathrm{R} / \mathrm{L}_{\mathrm{Te}}$, measured at $\mathrm{r} / \mathrm{a}=0.2$, is also maximum for $\mathrm{R}_{\mathrm{IC}}-\mathrm{R}_{\mathrm{mag}}=0.10-0.20 \mathrm{~m}$ and falls off very rapidly when the resonance layer is further out (Fig.4-b). The ion temperature gradient $\mathrm{R} / \mathrm{L}_{\mathrm{Ti}}$, measured at $\mathrm{r} / \mathrm{a} \sim 0.35$, also increases from $\sim 2$ to $\sim 4$ when $\mathrm{R}_{\mathrm{IC}}-\mathrm{R}_{\text {mag }}$ increases from $-0.15 \mathrm{~m}$ to $+0.20 \mathrm{~m}$. Surprisingly, the normalized logarithmic gradient of the density $\mathrm{R} / \mathrm{L}_{\mathrm{n}}$, increases monotonically from $\sim 0.5$ to $\sim 1.5$ when the IC resonance is moved from the HFS to the LFS. The sawtooth-free discharge has a slightly higher density gradient.
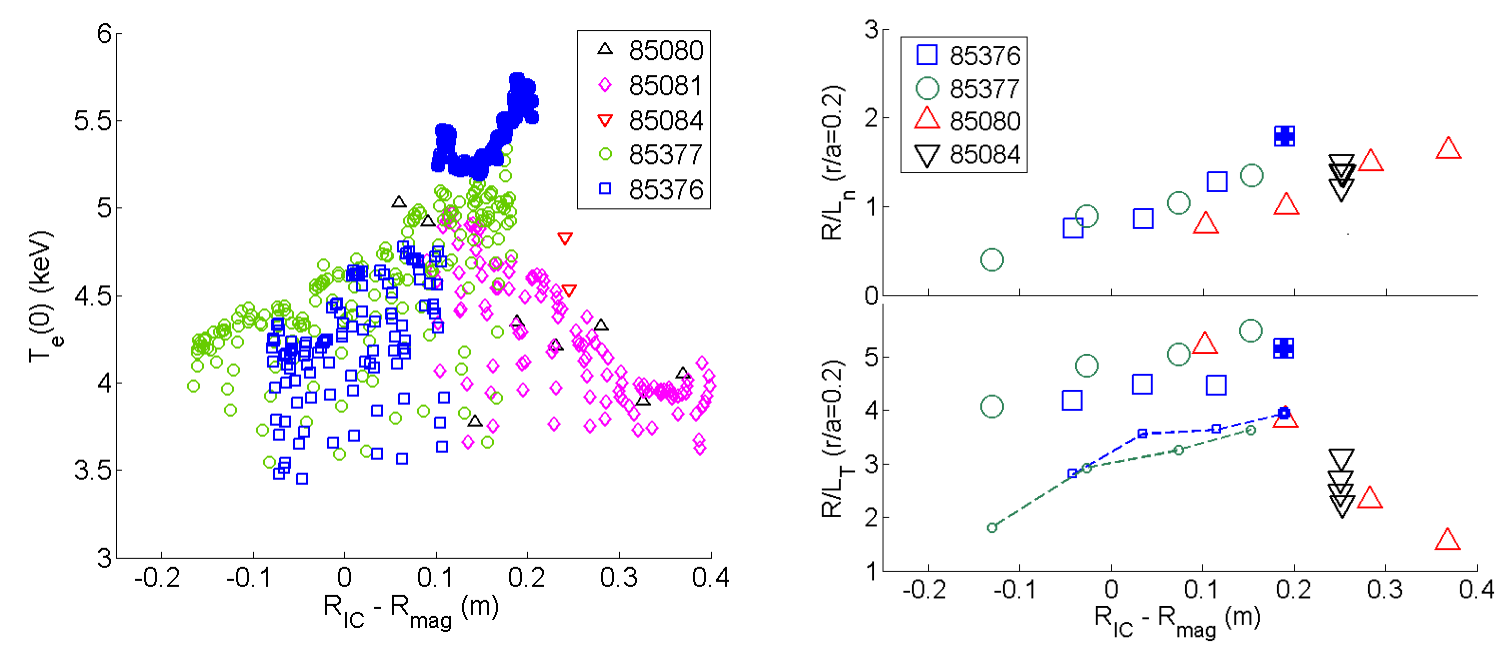

Figure 4 a) Central electron temperature b) Normalized density and temperature gradients as a function of the IC resonance layer position. Electron temperature (large symbols) and ion 
temperature gradients (small symbols, dashed lines) are measured at $r / a=0.2$ and 0.35 , respectively. The normalized gradients are obtained from profiles averaged on 1-second time slices. The close symbols are for the 1.3s long sawtooth-free period. The $q=1$ surface on the low field side is located at a distance from the magnetic axis $R_{q=1}-R_{\text {mag }} \approx 0.26 \mathrm{~m}$. The pulse 85084 ( $\nabla$ symbols) has constant $B / I_{p}$.
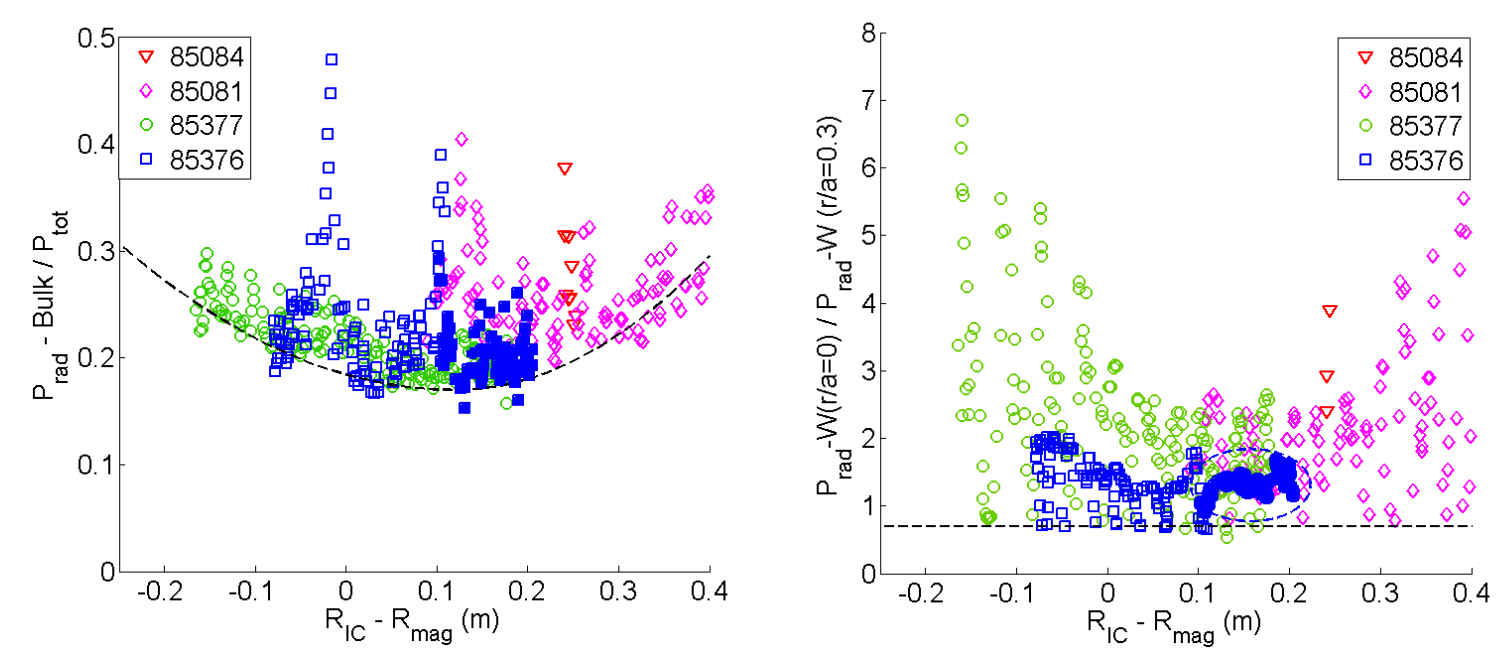

Figure 5 a) Fraction of the input power radiated in the main chamber b) Peaking of the radiated as a function of the IC resonance position. The close symbols are for the $1.3 \mathrm{~s}$ long sawtooth-free discharge. The pulse 85084 ( $\Delta$ symbols) has constant $B_{t} / I_{p}$.

The fraction of radiated power in the main chamber $\left(\mathrm{P}_{\mathrm{rad}}-\mathrm{Bulk} / \mathrm{P}_{\mathrm{tot}}\right)$ and the peaking of the tungsten radiation $\left(\mathrm{PF}_{0.3}=\mathrm{P}_{\mathrm{rad}-\mathrm{W}}(\mathrm{r} / \mathrm{a}=0) / \mathrm{P}_{\text {rad-W }}(\mathrm{r} / \mathrm{a}=0.3)\right.$ are both minimized when the IC resonance position is such that $\mathrm{R}_{\mathrm{IC}}-\mathrm{R}_{\text {mag }}$ lies between 0 and $0.20 \mathrm{~m}$ (figure 5 ). The absolute value of tungsten radiation at $\mathrm{r} / \mathrm{a}=0$ is also the lowest for this range of $\mathrm{IC}$ resonance position $\left(0.05-0.1 \mathrm{MW} / \mathrm{m}^{3}\right)$. After the sawtooth crash, the tungsten peaking drops to $\sim 0.7$ for any resonance position (dashed line of fig. 5-b). It is noticeable that despite a significant peaking of the density, the sawtooth free discharge has low radiation and low tungsten peaking ( 1.5). These quantities do not evolve significantly during this $1.3 \mathrm{~s}$ long phase of the discharge and are kept close to the minimum. The high temperature peaking $\left(\mathrm{R} / \mathrm{L}_{\mathrm{Te}} \approx 5\right.$ at $\mathrm{r} / \mathrm{a}=0.2, \mathrm{R} / \mathrm{L}_{\mathrm{Ti}} \sim 4$ at $\mathrm{r} / \mathrm{a}=0.35$ ) counterbalances the slight peaking of the density and the lack of sawteeth to flush out the tungsten. Sawtooth free discharge has also been obtained by reducing the hydrogen minority concentration to $\sim 2 \%$ and, although the core radiation 
increases very slightly, the core tungsten concentration is maintained below $10^{-4}$ for $3.5 \mathrm{~s}$ [Van Eester14, Goniche 14].

It should be noted that, at mid-radius $(\mathrm{r} / \mathrm{a}=0.5)$, tungsten as nickel concentration, derived from the NiXXV line intensity, is observed to vary by no more than $\pm 25 \%$ for these pulses.

It appears that by setting the resonance layer on the low field side, inside but near the $\mathrm{q}=1$ surface, the tungsten accumulation can be minimized in the baseline scenario. This is a quite favorable situation as ICRH can also be used for sawtooth control [Lennholm 15, Graves 15].

In addition to the sawtooth instability, other MHD modes are expected to act on the tungsten transport [Nave 2003, Pütterich 2013, Angioni 2014]. When the large sawtooth $(\Delta \mathrm{Te}=1.5 \mathrm{keV})$ occurs after the $1.3 \mathrm{~s}$ long sawtooth free period $(\# 85376), \mathrm{a}(\mathrm{m}=3, \mathrm{n}=2)$ neoclassical tearing mode (NTM) is destabilized for the rest of the discharge $(\mathrm{f}=9-10 \mathrm{kHz})$ whereas a $\mathrm{m}=1, \mathrm{n}=1$ kink mode $(\mathrm{f}=8-9 \mathrm{kHz})$ is triggered during the other pulses (\#85377 \&85081). This NTM does not seem to foster tungsten accumulation (tungsten peaking just increases transiently from $\sim 1.3$ to $\sim 1.7$ ) when for the same IC resonance position, the tungsten peaking of the pulse with the $(1,1)$ mode $(\# 85377)$ has indeed a higher tungsten peaking. In both cases, fishbone modes with a quite large frequency span $(f=5-9 \mathrm{kHz}$ for $85376, \mathrm{f}=7-11 \mathrm{kHz}$ for $85377, \mathrm{f}=8-13 \mathrm{kHz}$ for 85081 ) are destabilized when the IC position is on the LFS, close to the magnetic axis $\left(0<\mathrm{R}_{\mathrm{IC}}-\mathrm{R}_{\mathrm{mag}}<0.15 \mathrm{~m}\right)$ (figure 6$)$. 

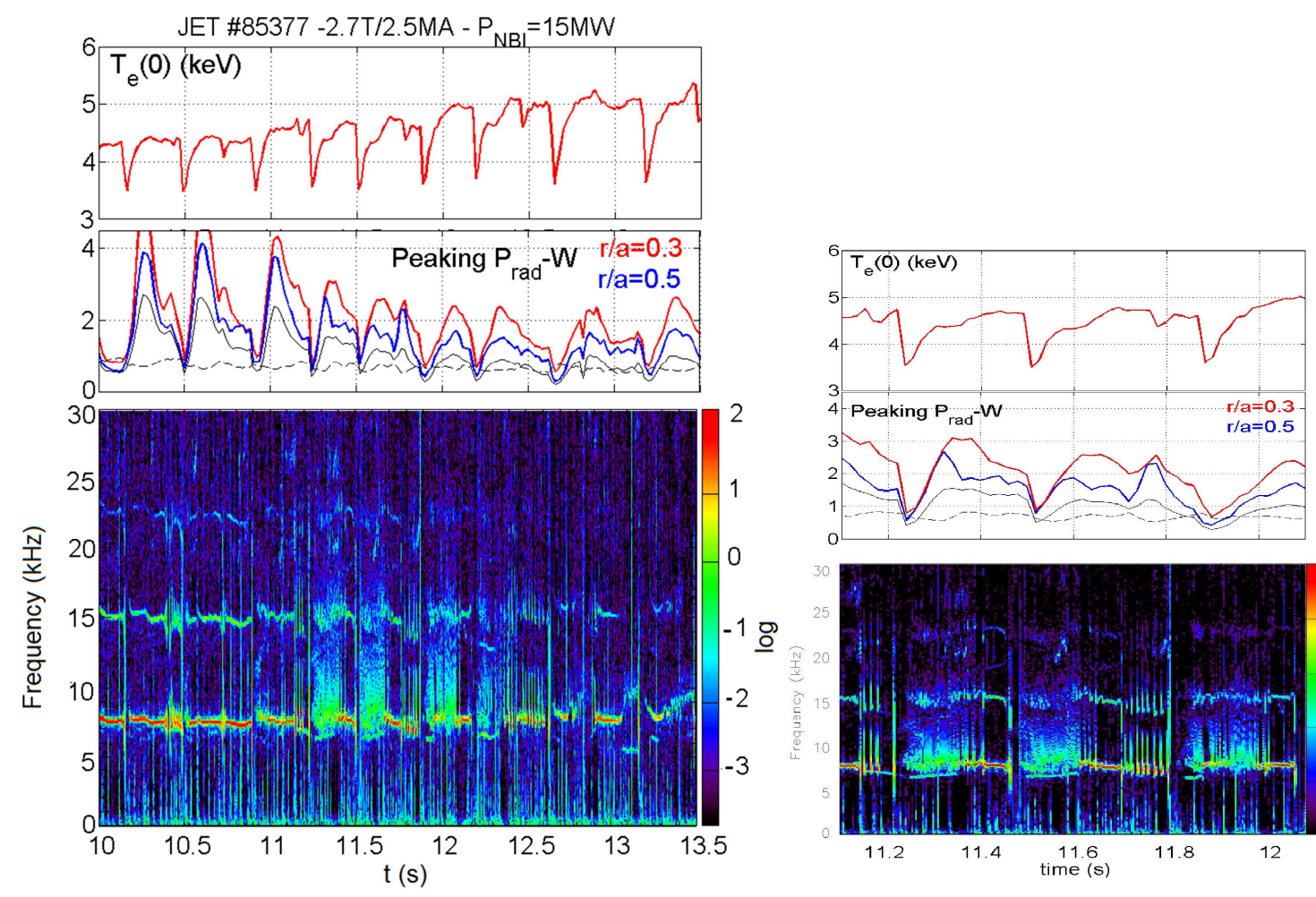

Figure 6. Central temperature, tungsten radiation peaking $P_{r a d}(r / a=0) / P_{r a d}(r / a=0.3)$ (red line), $P_{r a d}(r / a=0) / P_{r a d}(r / a=0.5)$ (blue line), tungsten radiation $P_{r a d}(r / a=0)\left(10^{5} \mathrm{~W} / \mathrm{m}^{3}\right.$, black solid line), $P_{\text {rad }}(r / a=0.5)\left(10^{5} \mathrm{~W} / \mathrm{m}^{3}\right.$, black dotted line), spectrogram (from magnetic loops). Magnetic field is ramped up during this discharge. $R_{I C}-R_{\text {mag }}=0$ at $t=11.0 \mathrm{~s}$ and $R_{I C}-R_{\text {mag }}=0.13 \mathrm{~m}$ at $t=12.5 \mathrm{~s}$ $\left(P_{\text {ICRH }}=4 M W\right)$.

These fishbone activity, which does not last more than $100-150 \mathrm{~ms}$, alternate with the kink mode which should be the result of a modulation of the fast ion pressure gradient from high (just after the sawtooth crash) to lower value (ref.?). In that case, core tungsten radiation has actually a 3-step cycle. Starting from the sawtooth crash, there is a fast and short flattening of the radiation profile, followed by peaking lasting $100-200 \mathrm{~ms}$ and finally the core profile flattens again for 200-300ms until the next sawtooth crash (figure 6).

\section{ICRH in JET Baseline scenario}

\subsection{Medium plasma current experiments}

ICRH power varying between 0 and $6 \mathrm{MW}$ was coupled to $2.7 \mathrm{~T} / 2.5 \mathrm{MA}$ discharges with total power close to $20 \mathrm{MW}$ at a frequency of $42.5 \mathrm{MHz}$ providing central heating with 
the IC resonance layer located about $5 \mathrm{~cm}$ from the magnetic axis on the high field side in most cases.

The first series was performed with the outer strike point on the tile 5 of the bottom divertor $\left(\mathrm{R}_{\mathrm{OSP}} \sim 2.73 \mathrm{~m}\right)$ and gas rate in the range of $0.9-1.2 \times 10^{22} \mathrm{el} . / \mathrm{s}$. This allows to be well above the L-H transition and to get type I ELMs with frequency in the $30-45 \mathrm{~Hz}$ range. For the same total power (19-20MW), when the ICRH power is increased from 0 to $6 \mathrm{MW}$, a strong decrease of the tungsten radiation in the very core $(\mathrm{r} / \mathrm{a} \sim 0)$ from $0.2 \mathrm{MW} / \mathrm{m}^{3}$ to $0.07 \mathrm{MW} / \mathrm{m}^{3}$ is observed and the corresponding peaking from $\sim 10$ to $\sim 1.5$ (figure $7-\mathrm{a}$ ). A weaker decrease is observed further out in the plasma volume and at r/a 0.45, radiation pet unit of volume starts increasing and slowly decreases when the ICRH power exceeds 4$5 \mathrm{MW}$. The same trend is observed for the nickel concentration and for the total radiated power from bolometry. The LFS/HFS asymmetry of the SXR radiation can be estimated from the ratio of the raw signals given by the lines of sight tangent to the same magnetic surface. This was done at $\mathrm{r} / \mathrm{a}=0.3$ and interpolation from the 2 nearest lines of sight was carried out for better accuracy (figure $7-b)$. This asymmetry decreases from $1.6\left(\mathrm{P}_{\text {ICRH }}=0\right)$ to $1.1\left(\mathrm{P}_{\text {ICRH }} \sim 6 \mathrm{MW}\right)$, demonstrating the beneficial effect of the ICRH on the asymmetry of the radiation. As an effect of reduced contamination of the plasma core in high- $Z$ impurities, energy confinement, evaluated from the $\mathrm{H}_{98 \mathrm{y}, 2}$ factor, slightly increases from $\sim 0.72$ to $\sim 0.77$ after correction of ICRH fast ion contribution to the plasma pressure.
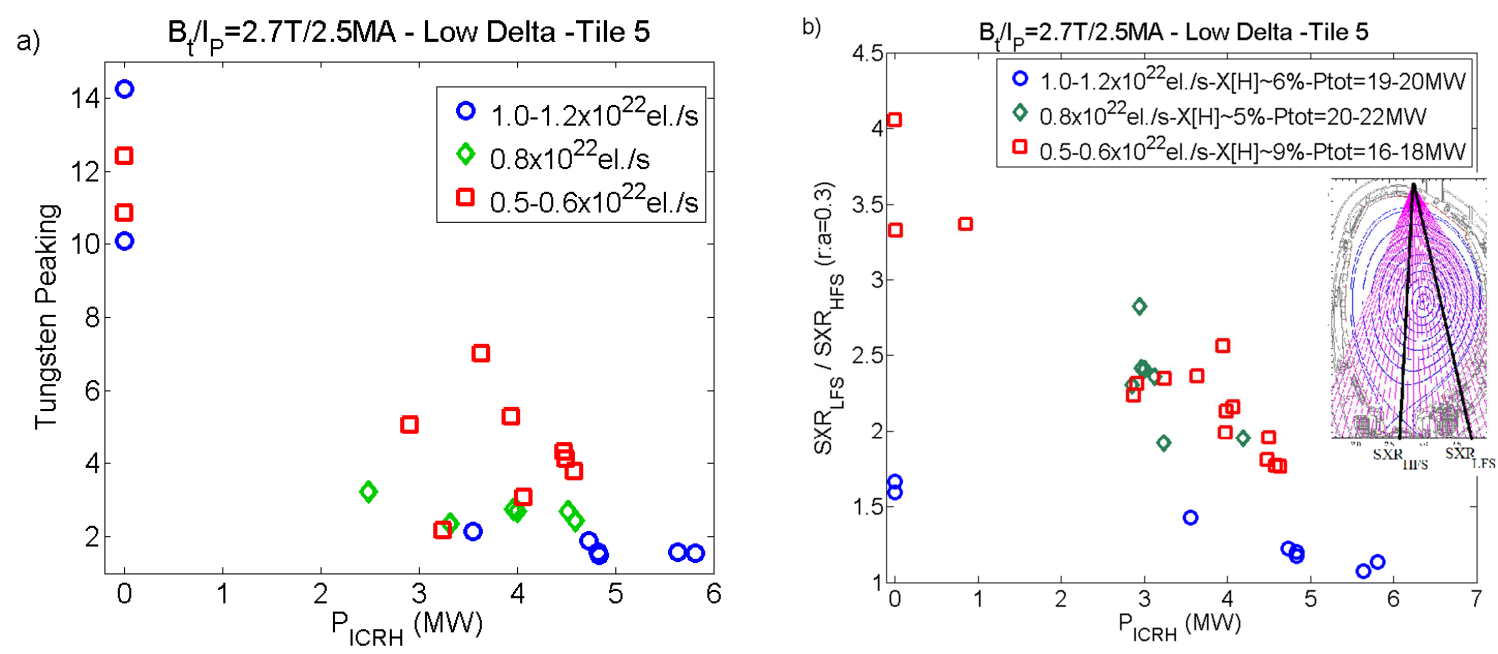

Figure 7 a) Radiated powers (total from the bulk of the plasma and from tungsten at different radii, flux surface-averaged). b) asymmetry of the SXR radiation in the mid-plane at r/a=0.3. Nearest tangent line-of-sight is chord 13 (HFS) and 23 (LFS). Data collected at least 2seconds after the start of the high power phase 
At low gas rate $\left(\sim 0.5 \times 10^{22} \mathrm{el} . / \mathrm{s}\right)$, ELM frequency decreases to $15-20 \mathrm{~Hz}$ and both the total radiation and core tungsten radiation increase strongly. $\mathrm{P}_{\mathrm{rad}}-\mathrm{bulk} / \mathrm{P}_{\text {tot }}$ is still above $45 \%$ and $\mathrm{P}_{\mathrm{rad}}-\mathrm{W}(\mathrm{r} / \mathrm{a}=0)$ above $0.4 \mathrm{MW} / \mathrm{m}^{3}$ with $4.6 \mathrm{MW}$ of ICRH power. However the SXR radiation asymmetry decreases linearly with power from $\sim 4\left(\mathrm{P}_{\mathrm{ICRH}}=0\right)$ to $\sim 1.8\left(\mathrm{P}_{\mathrm{ICRH}}=4.6 \mathrm{MW}\right)$ (figure 7-b).

When comparing, the progress of a 4-s long high power phase with low $\left(0.5 \times 10^{22} \mathrm{el} . / \mathrm{s}\right)$ and medium $\left(1.1 \times 10^{22} \mathrm{el} . / \mathrm{s}\right)$ gas rate and same ICRH power $(\sim 4.6 \mathrm{MW})$, these discharges have same sawtooth period $(\sim 380 \mathrm{~ms})$ and slightly higher central electron temperature (due to higher NBI power) for the medium gas dosing case (Figure 8). The tungsten is expelled from the core $(r / a<0.2)$ during the sawtooth crash and a quasi-periodic regime is established for the tungsten radiation with no trend of further increase on a long time scale. It should be noted that, although the central radiation is higher by a factor $\sim 6$ and the total radiation by a factor 2.5 for the low gas dosing discharge, the confinement is improved and the $\mathrm{H}$-factor increases from $\sim 0.79$ to $\sim 0.85$, thanks to the higher pedestal pressure.

When the outer strike point is moved closer to the pumping duct ( $\left.\mathrm{R}_{\mathrm{OSP}} \sim 2.92 \mathrm{~m}\right)$, with higher gas dosing $\left(1.7 \times 10^{22} \mathrm{el} . / \mathrm{s}\right)$, high ELM frequency $(\sim 100 \mathrm{~Hz})$ is combined with low radiation $\left(\mathrm{P}_{\text {rad } /} \mathrm{P}_{\text {tot }} \sim 20 \%, \mathrm{P}_{\text {rad }}-\mathrm{W}(0) \sim 0.06 \mathrm{MW} / \mathrm{m}^{3}\right)$ and similar confinement to that of the low gas dosing case $\left(\mathrm{H}_{98, \mathrm{y}} \sim 0.85\right)$. 


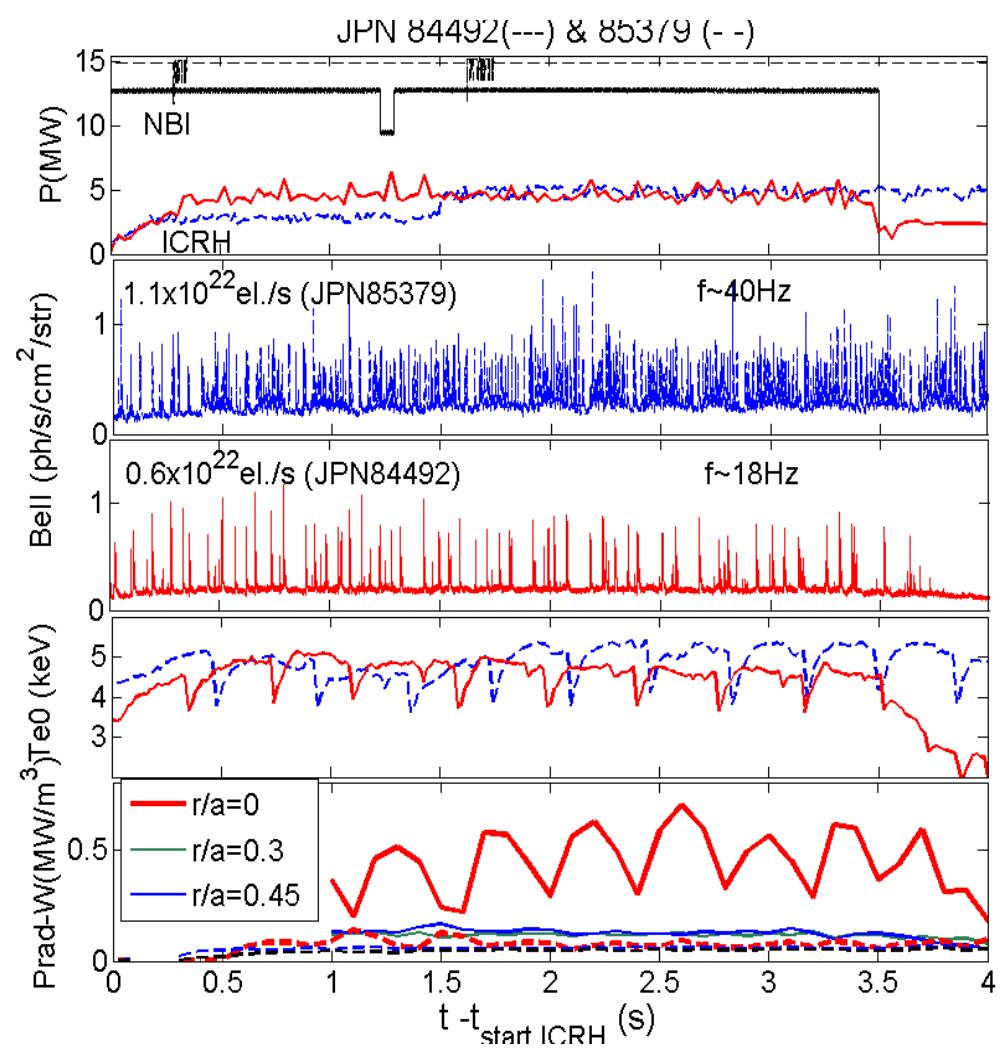

Figure 8. Effect of gas rate on ELMs frequency, $T_{e}(0)$ and tungsten radiation (from SXR diagnostic). $B_{t}=2.7 T, I_{p}=2.5 M A, n_{e}(0)=7.1 \times 10^{19} \mathrm{~m}^{-3}$.

When the global confinement properties depend on the level of impurities radiation which combine effect of the source, partially depending on the ELMs amplitude (and consequently on the ELM frequency) and the particle transport, insight on this transport can be addressed from the density gradients of these species.

For all gas dosing and pumping cases, the normalized logarithmic gradient of the electron temperature $\mathrm{R}\left(\mathrm{dT}_{\mathrm{e}} / \mathrm{dR}\right) / \mathrm{T}_{\mathrm{e}}=\mathrm{R} / \mathrm{L}_{\mathrm{Te}}$, increases from $\sim 2$ to $\sim 5$ when the ICRH power increases from 0 to $6 \mathrm{MW}$ (figure 9). The equivalent quantity for the density, $\mathrm{R} / \mathrm{L}_{\mathrm{n}}$, decreases with the ICRH power from $\sim 1.5$ to $\sim 0.5$ for the low pumping case. In the case of high pumping and high gas dosing, the central density is significantly lower $\left(\mathrm{n}_{\mathrm{e}}(0) \sim 6 \times 10^{19} \mathrm{~m}^{-3}\right)$ compared to the low pumping case $\left(\mathrm{n}_{\mathrm{e}}(0) \sim 7 \times 10^{19} \mathrm{~m}^{-3}\right)$ with $4 \mathrm{MW}$ of ICRH power and the normalized logarithmic gradient is higher $\left(\mathrm{R} / \mathrm{L}_{\mathrm{n}} \sim 2\right)$. 


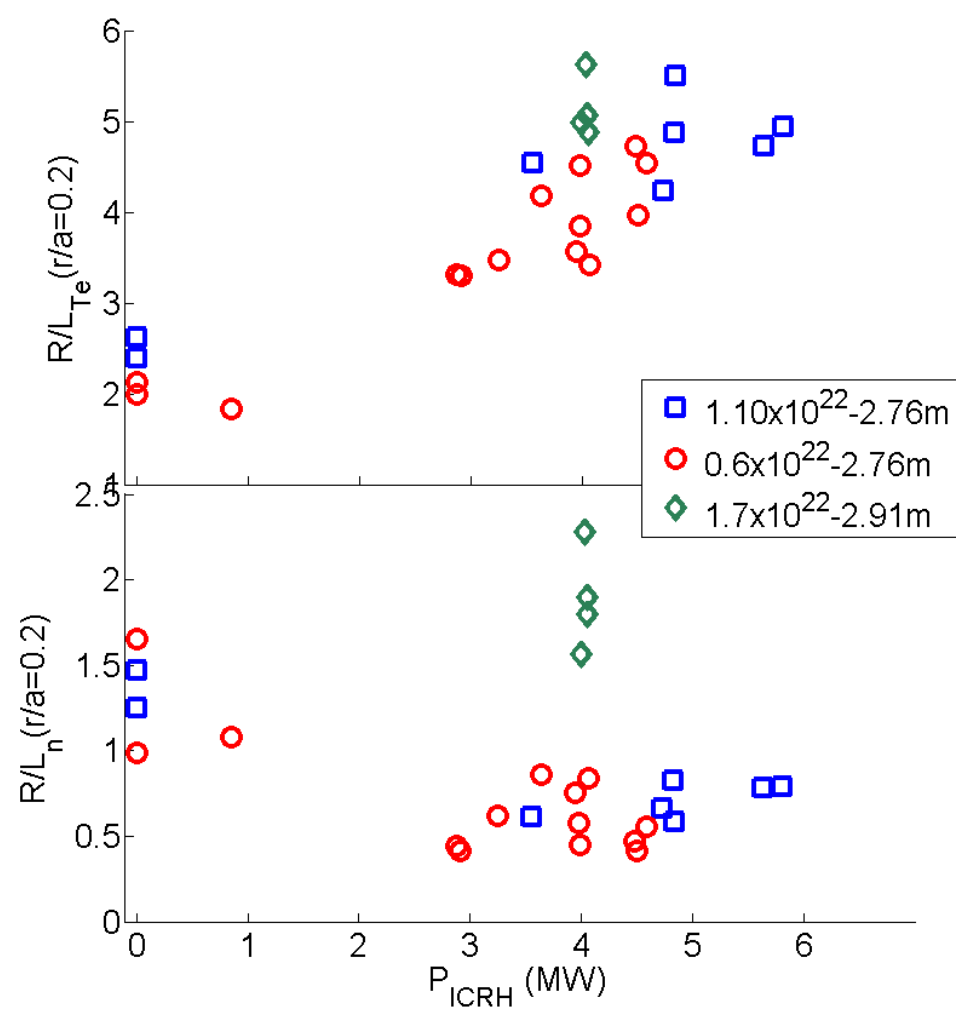

Figure 9. Normalized gradient lengths at r/a 0.2 for various gas rates $\left(0.6,1.1\right.$ and $1.7 \times 10^{22}$ el./s), and strike point positions on the outer divertor $(2.76 \mathrm{~m}$ and $2.91 \mathrm{~m})$.Total power is in the $18-20 \mathrm{MW}$ range. The ELM frequency is $15-20,30-45,90-100 \mathrm{~Hz}$ for, respectively, the 0.6, 1.1, $1.7 \times 10^{22}$ el./s cases.

The ion temperature in the plasma core $(\mathrm{r} / \mathrm{a}<0.3)$ are not available for these discharges and the tungsten flux is estimated from $R / \mathrm{L}_{n}-0.5 \mathrm{R} / \mathrm{L}_{\mathrm{T}}$ assuming that $\mathrm{n}_{\mathrm{i}}=\mathrm{n}_{\mathrm{e}}$ and $\mathrm{T}_{\mathrm{i}}=\mathrm{T}_{\mathrm{e}}$. This last assumption can be questionable although ICRH modeling (see section 2) has shown than even at moderate densities $\left(n_{e}(0)=6.5 \times 10^{19} \mathrm{~m}^{-3}\right)$, the RF power transferred to the electrons and ions do not departure from equipartition by much after collisional redistribution. When comparing the low density/low gas dosing discharge (circle symbols of figure 9) to the high gas dosing discharge (diamond symbols) with the same ICRH power $(4 \mathrm{MW}), \mathrm{R} / \mathrm{L}_{\mathrm{ne}}-$ $0.5 \mathrm{R} / \mathrm{L}_{\mathrm{Te}}$ is negative, close to -1 , for the former and close to -0.5 for the latter. However, the tungsten peaking evaluated from $\mathrm{PF}_{0.3}$ is found to be quite high $\left(\mathrm{PF}_{0.3}=2-7\right)$, for the low gas rate case and low $\left(\mathrm{PF}_{0.3}=1.3-2\right)$ for the high gas rate case.

The tungsten radiation peaking is estimated on a large data base including pulses with various $\mathrm{P}_{\text {tot }}(15-20 \mathrm{MW}), \mathrm{P}_{\mathrm{ICRH}}(0-6 \mathrm{MW})$, minority hydrogen concentration $\mathrm{X}[\mathrm{H}](2-20 \%)$. It 
is found a good correlation between $\mathrm{R} / \mathrm{L}_{\mathrm{ne}}-0.5 \mathrm{R} / \mathrm{L}_{\mathrm{Te}}$ and the tungsten peaking which is varying between 1 and 14 (figure 10). Beneficial effect of ICRH on the gradients is clearly seen and sign of convection is inverted from inward to outward for an ICRH power exceeding 3MW for most cases. However, as mentioned in previous paragraph, a significant difference is observed between low gas rate/low pumping pulses (+ symbols of figure 10) and high gas rate/high pumping pulses ( $\times$ symbols of figure 10$)$ : although they have almost the same $\mathrm{R} / \mathrm{L}_{\mathrm{ne}}-0.5 \mathrm{R} / \mathrm{L}_{\mathrm{Te}}$ value, higher peaking of the tungsten radiation is observed for the low gas rate pulses. In that case we expect a higher tungsten source from the edge resulting from the lower ELM frequency but also from the higher rectified electric field, caused by the weaker coupling (the coupling resistance is lower by 20-25\%). Consistently, the core tungsten radiation is about 10 times higher when compared to the high gas rate cases.

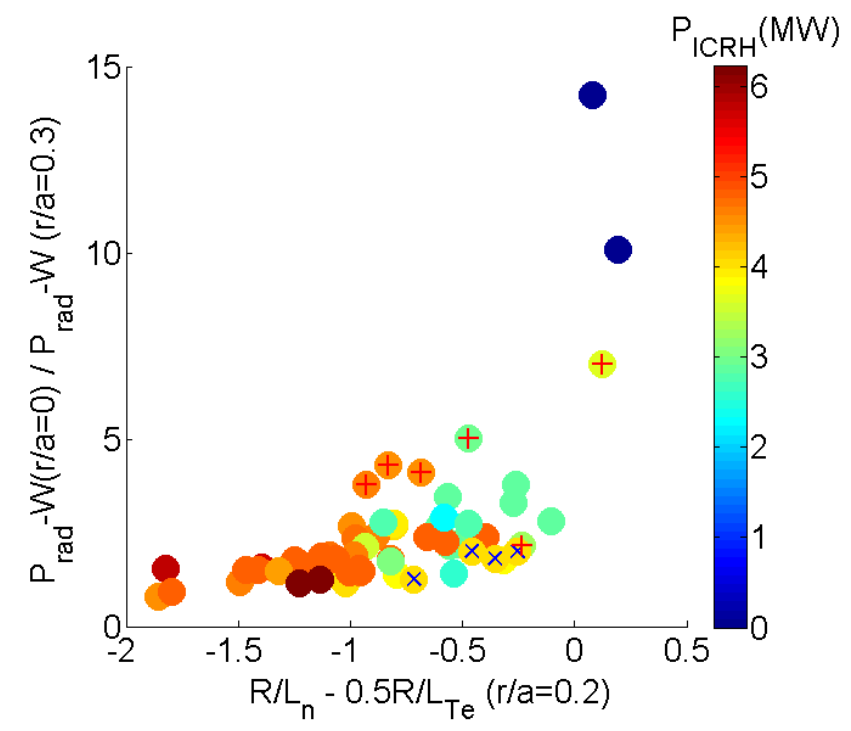

Figure 10. Tungsten radiation peaking (from SXR measurements) as a function of $R / L_{n e}-0.5 R / L_{T e}$. Electron densities are provided by the LIDAR diagnostic, the electron temperature by the ECE diagnostic. Low gas rate/low pumping $(+)$ and high gas rate/high pumping $(\times)$ cases of figure 7 are highlighted. Data are averaged on a 1-second time window, at least 1 second after the start of the ICRH power. $B t=2.7 T, I p=2.5 M A$.

\subsection{High Plasma current experiments}

For the development of the baseline scenario, in preparation of the D-T campaign, the plasma current was increased from 2.5 to $4.0 \mathrm{MA}$ while keeping $\mathrm{q}_{95}$ close to $3\left(\mathrm{~B}_{\mathrm{t}}=2.7-3.7 \mathrm{~T}\right)$ [Nunes 2014]. The outer strike point is close to the pumping duct ( $\left.\mathrm{R}_{\mathrm{OSP}} \sim 2.92 \mathrm{~m}\right)$ and the gas injection rate is increased with respect of the lower plasma experiments $\left(2-5 \times 10^{22} \mathrm{el} . / \mathrm{s}\right)$. Total power was varied between $15 \mathrm{MW}$ and $30 \mathrm{MW}$ and the ICRH power in the $2-5 \mathrm{MW}$ 
range. The position of the IC resonance layer is constrained by the scenario and the available bands of frequency of the ICRH generators. As a consequence the position of the IC resonance layer was varied such as $0<\mathrm{R}_{\mathrm{IC}}-\mathrm{R}_{\mathrm{mag}}<0.50 \mathrm{~m}$.

At 3.0MA with medium gas injection $\left(\sim 3 \times 10^{22} \mathrm{el} . / \mathrm{s}\right)$, allowing to have a high ELM frequency $(\sim 100 \mathrm{~Hz})$, the maximum core tungsten peaking increases regularly from one sawtooth cycle to the next one (figure 11-a), exceeding 4, 3 seconds after the start of the ICRH power $\left(\mathrm{P}_{\text {ICRH }}=4.5 \mathrm{MW}\right)$. The $\mathrm{W}$ peaking (and the absolute radiated power at the center) is also strongly modulated with the sawtooth period but the flat tungsten profile (peaking =1) is achieved now just before the sawtooth crash and the 3-step cycle described for $2.5 \mathrm{MA}$ discharges (see section 2) is now a 2-step cycle where the peaking increases with Te after the sawtooth crash and decreases, until the next sawtooth crash, when $\mathrm{T}_{\mathrm{e}}$ is stationary or even slightly decreasing as observed between $\mathrm{t}=9.7$ (resp. 10.25s) and 10.1s (resp.10.65s). The peaking increase is accompanied by fishbone activity with low amplitude and a large frequency span (between 10 and $15 \mathrm{kHz}$ ). This is followed, during the $\mathrm{W}$ radiation flattening phase, by fishbones with smaller frequency span and large amplitude. The $(1,1)$ mode alternating with the fishbones is growing in amplitude until the next sawtooth.

For a pulse performed with the same plasma current, same NBI and ICRH powers but with higher magnetic field and safety factor $\left(\mathrm{q}_{95}=3.6\right)$ and about the same ELM frequency $(\sim 80 \mathrm{~Hz})$, a slightly different MHD activity is observed during a sawtooth period. During the radiation peaking phase, fishbones with large frequency span are still observed but this activity is triggered later and the amplitude is smaller. Later, the tungsten flattening is weaker and the amplitude of the fishbones reduced with respect of the low $\mathrm{q}_{95}$ discharge. The same modulation of the tungsten radiation peaking during the sawtooth period is observed with about the same maximum between sawteeth but a flat profile (peaking factor $=1$ ) is only obtained when the sawtooth crashes (figure 11-b). This discharge has no or very weak $(1,1)$ mode for most of the sawtooth cycles. However it should be pointed out that the plasma stored energy (from diamagnetic measurements) is identical for the two discharges (within $5 \%$ ), but the $\mathrm{H}$ factor $(\mathrm{H} 98 \mathrm{y})$ is significantly higher for the low $\mathrm{q}_{95}$ discharge (0.82 vs. 0.76). 

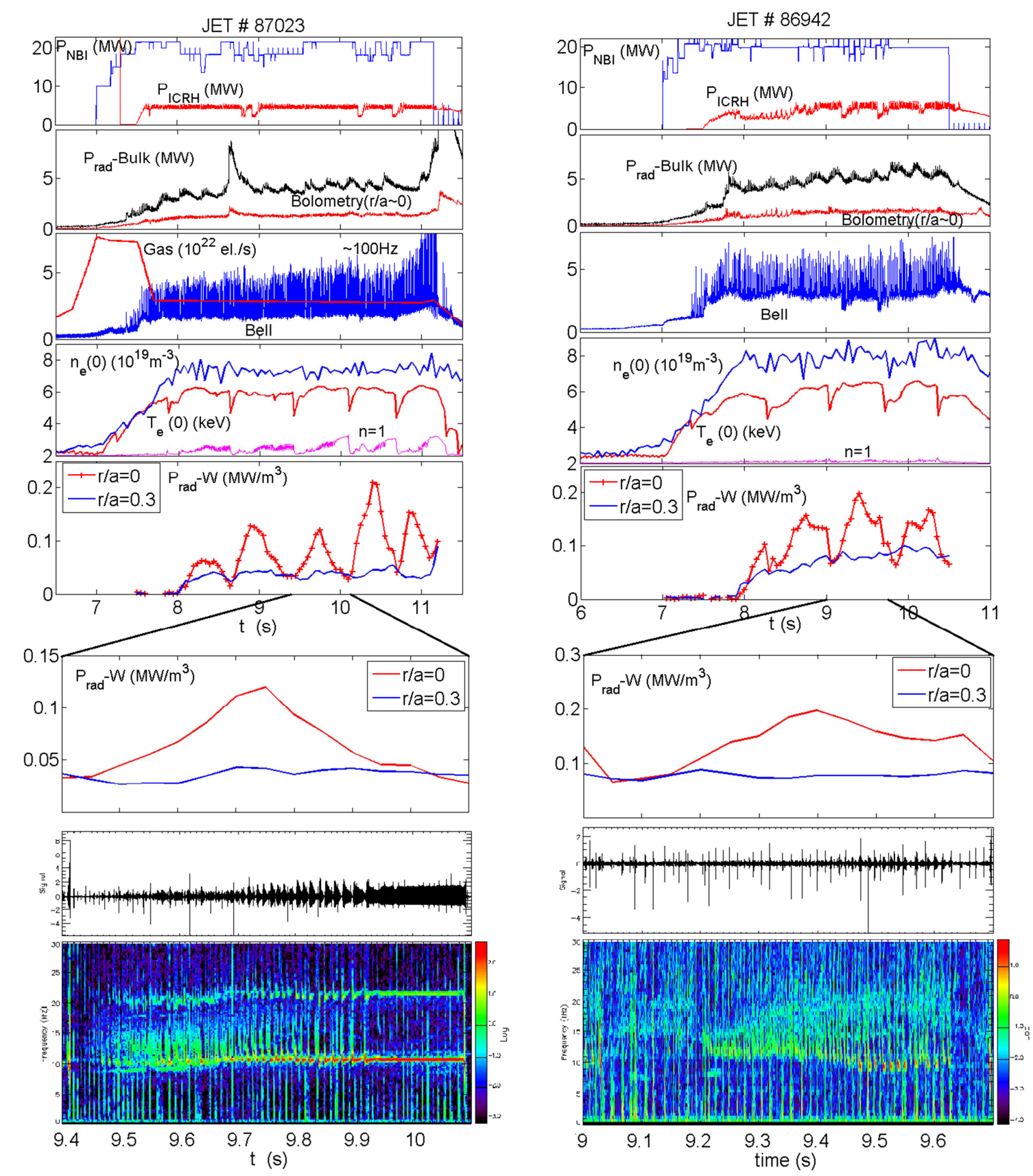

Figure 11. Time traces of 3MA discharge a) $87023\left(q_{95}=3.0\right)$ b) $86942\left(q_{95}=3.65\right)$. For both pulses: $P_{N B I} \approx 20 M W, P_{I C R H} \approx 4.5 M W, R_{I C} R_{m a g}=0.10 m, X[H] \approx 5 \%$. A blow-up of the tungsten radiation between two sawtooth crashes and the corresponding spectrogram from a magnetic pick-up coil are displayed in the bottom of the figures. The scale of the spectrogram of discharge 86942 is reduced by a factor 10 .

When the IC resonance is moved out of the $q=1$ surface $\left(R_{I C}-R_{m a g}=0.39 m\right)$, although the gas rate is strongly increased $\left(\sim 5 \times 10^{22} \mathrm{el} . / \mathrm{s}\right)$, to maintain a high ELM frequency $(\sim 100 \mathrm{~Hz})$, peaking of the tungsten radiation now increases to very high values (10-20) until the sawtooth crashes when the radiation profile gets flat with a peaking close to 1 (figure 
12). No fishbone activity, but a strong continuous $(1,1)$ mode, is triggered by ICRH fast ions as the trapped ions are now mostly out of $\mathrm{q}=1$ surface.

It should be noted that, when comparing discharges with same $\mathrm{q}_{95}$ but resonance inside (\#87023) and outside (\#87380) the $\mathrm{q}=1$ surface, the tungsten radiation at the center (r/a 0) just increases from $\sim 0.1 \mathrm{MW} / \mathrm{m}^{3}$ to $\sim 0.15 \mathrm{MW} / \mathrm{m}^{3}$ when this quantity is averaged on a sawtooth period. The total powers radiated in the main chamber are also very similar with $\mathrm{P}_{\mathrm{rad}}-\mathrm{Bulk} / \mathrm{P}_{\text {tot }} \sim 0.16-0.18$, which are low ratios with respect of those obtained at $2.5 \mathrm{MA}$ for which $\mathrm{P}_{\mathrm{rad}}-\mathrm{Bulk} / \mathrm{P}_{\text {tot }} \sim 0.18-0.21$ The density normalized gradients are very similar for these pulses $\left(R / L_{n} \sim 1\right)$ but the temperature gradient is higher when the resonance is central $\left(\mathrm{R}_{\mathrm{L}} / \mathrm{L}_{\mathrm{Te}} \sim 4\right.$ vs 2.5$)$. Ion temperature, measured at $\mathrm{r} / \mathrm{a} \sim 0.4$, is also much higher (factor $\sim 1.4$ ), in rough agreement with the neutron yield (factor $\sim 1.6$ ), for the central heating case. It results a $\mathrm{R} / \mathrm{L}_{\mathrm{ne}}-0.5 \mathrm{R} / \mathrm{L}_{\mathrm{Te}}$ value lower for central heating ( - 1$)$ than for off-axis heating $(\sim 0)$ when the average tungsten peaking varies between 2 and 3 for the first case and 6 and 7 for the second one. These data (peaking vs $\mathrm{R} / \mathrm{L}_{\mathrm{ne}}-0.5 \mathrm{R} / \mathrm{L}_{\mathrm{Te}}$ ) fit those obtained at $2.5 \mathrm{MA}$ with low gas rate /low pumping (+ symbols of figure 10).

When the current is further increased to $3.5 \mathrm{MA}$ with quite low gas injection $(\sim 2$ $\left.\times 10^{22} \mathrm{el} . / \mathrm{s}\right)$ and low ELM frequency $(\sim 40 \mathrm{~Hz})$, the radiated power increases strongly $\left(\mathrm{P}_{\mathrm{rad}}{ }^{-}\right.$ Bulk/ $\mathrm{P}_{\text {tot }} \sim 0.4-0.45$ ) and the maximum tungsten peaking increases during the 3-second high power phase up to $\sim 10$. At the same time the core tungsten radiation exceeds $0.7 \mathrm{MW} / \mathrm{m}^{3}$. The maximum of $\mathrm{W}$ radiation peaking (and of maximum core radiation) is now observed only $\sim 100 \mathrm{~ms}$ after the sawtooth crash $\left(\mathrm{t}_{\mathrm{ST}} \sim 600 \mathrm{~ms}\right)$. Off-axis ICRH heating $\left(\mathrm{R}_{\mathrm{IC}^{-}}\right.$ $\left.\mathrm{R}_{\mathrm{mag}}=0.40 \mathrm{~m}\right)$, with higher gas injection $\left(\sim 3.5 \times 10^{22} \mathrm{el} . / \mathrm{s}\right)$ and slightly higher ELM frequency $(\sim 50 \mathrm{~Hz})$ leads to a strongly peaked tungsten profile, with a peaking factor exceeding 10 , just before the sawtooth crash as observed at a plasma current of 3MA. The normalized density and temperature gradient lengths, averaged on one second, both flatten weakly when going from 3.0MA to 3.5MA and the $\mathrm{R} / \mathrm{L}_{\mathrm{ne}}-0.5 \mathrm{R} / \mathrm{L}_{\mathrm{Te}}$ quantity is unchanged ( - 1$)$ for the central ICRH heating scenario. This is roughly consistent with the $\mathrm{W}$ radiation peaking, averaged on one second, which increases only from $\sim 2$ to $\sim 3,1.5$ s after the start of the ICRH power. In the case of the off-axis ICRH, R/L $/ \mathrm{L}_{\mathrm{ne}}-0.5 \mathrm{R} / \mathrm{L}_{\mathrm{Te}} \sim 0$ for the two values of plasma current when the averaged $\mathrm{W}$ radiation peaking is $\sim 9$ at $3 \mathrm{MA}$ and $\sim 5$ at $3.5 \mathrm{MA}, 2 \mathrm{~s}$ after the start of the ICRH power. 


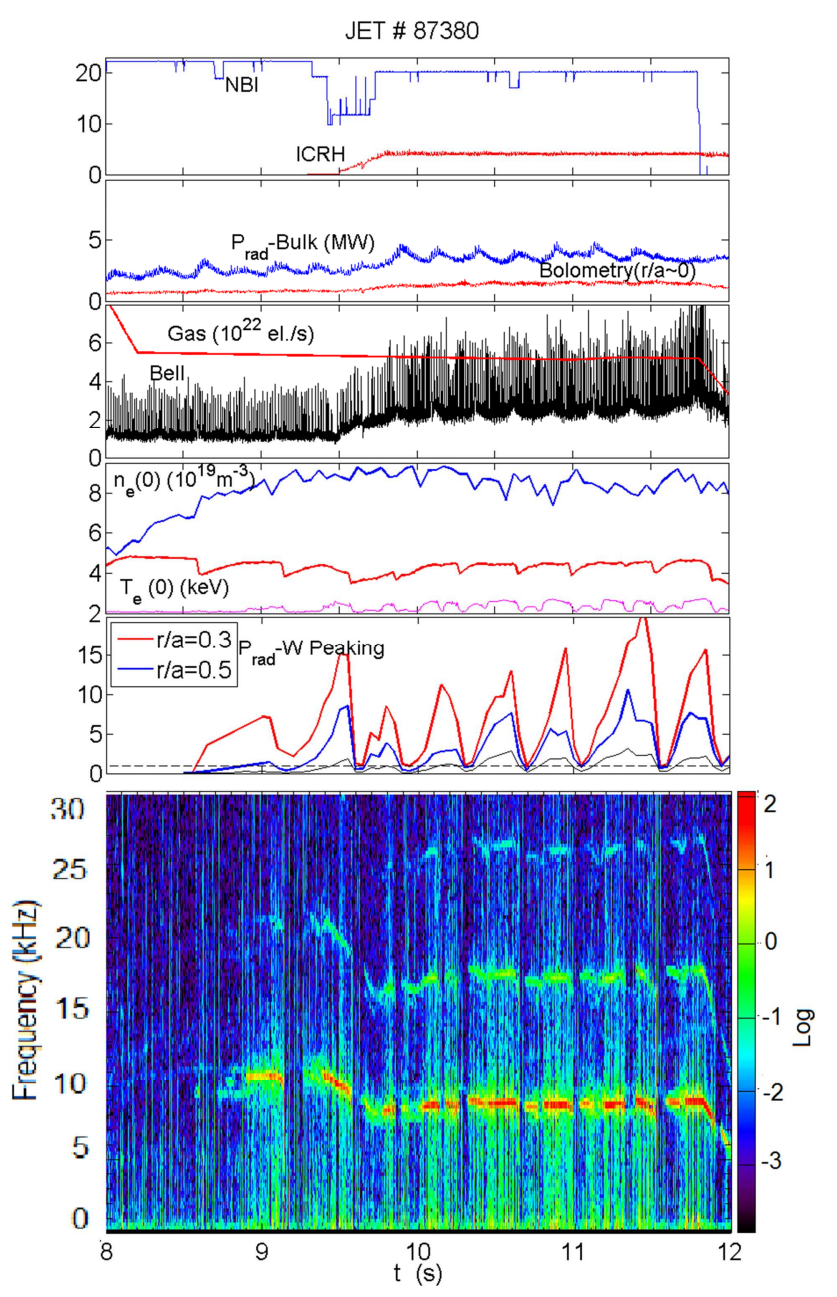

Figure 12 Time traces of $3 M A$ discharge $87380\left(q_{95}=3.0\right)$ with $P_{N B I} \approx 20 M W, P_{I C R H} \approx 4 M W, R_{I C \text { - }}$ $R_{\text {mag }}=0.39 m, X[H] \approx 7 \%$.

As a conclusion, when the current is increased from 2.5MA to 3.5MA with $\mathrm{q}_{95} \sim 3$, the maximum of tungsten peaking increases after 2-3 seconds of high power phase $\left(\mathrm{P}_{\text {tot }} \sim 24-\right.$ $27 \mathrm{MW})$ with central ICRH heating $\left(\mathrm{P}_{\mathrm{ICRH}} \sim 4 \mathrm{MW}\right)$, from $\sim 2$ to $\sim 10(\sim 4$ at $3 \mathrm{MA})$. At the same time, core $(\mathrm{r} / \mathrm{a} \sim 0)$ tungsten radiation increases from $\sim 0.1 \mathrm{MW} / \mathrm{m}^{3}\left(\mathrm{n}_{\mathrm{W}} \sim 10^{-4}\right)$ to $\sim 1 \mathrm{MW} / \mathrm{m}^{3}$ $\left(\mathrm{n}_{\mathrm{W}} \sim 10^{-3}\right)$. Tungsten peaking and core tungsten radiation are the highest after the sawtooth crash and decrease later until the next sawtooth crash. This increase and decrease of tungsten peaking is correlated with fishbone activity as follows. In the peaking phase, modes with chirping frequency typical of fishbone activity when the fast ion pressure gradient is large are detected [Breizman 2011]. During the flattening phase, this fishbone activity gets weaker, a continuous frequency $(\sim 10 \mathrm{kHz})$ mode, identified as the $(1,1)$, alternating with the weak fishbones, grows and eventually the fishbone activity vanishes. This gap mode is 
typical of low fast ion pressure gradient. When the IC resonance is moved out of the $q=1$ surface on the low field side, the amplitude of the sawtooth activity is reduced, the fishbone activity is suppressed, but a strong $(1,1)$ mode accompanies the strong increase of the tungsten peaking which now exceeds 10 very shortly after the start of the high power phase. Beneficial effect of ICRH above 3-4MW on tungsten peaking is confirmed. However, at moderate gas injection $\left(1-2 \times 10^{22} \mathrm{el} . / \mathrm{s}\right)$, there is a significant increase of the tungsten peaking at high plasma current $(\mathrm{Ip}=3.0-4.0 \mathrm{MA})$ with respect of the peaking obtained at lower current $(\mathrm{Ip}=2.5 \mathrm{MA})$ (figure 13).

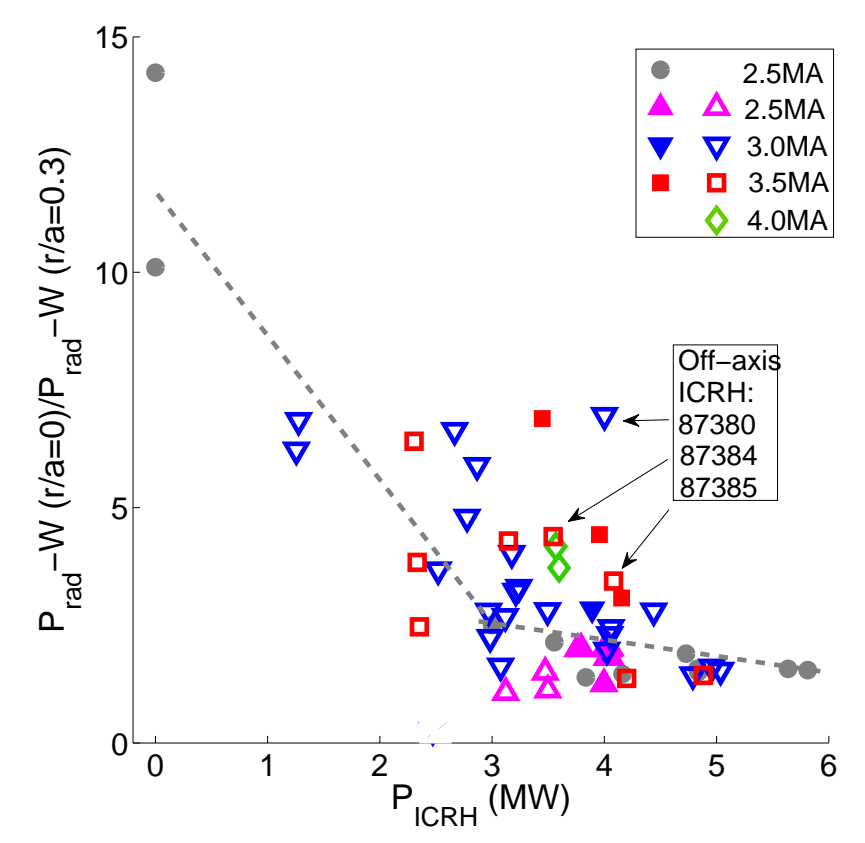

Figure 13. Tungsten radiation peaking at various plasma currents and ICRH powers. Closes symbols are for low to medium gas rate discharges $\left(1-2 \times 10^{22}\right.$ el./s, open symbols for high gas rate discharges $\left(2.3-5 \times 10^{22}\right.$ el./s). Low pumping discharges (see figure 7$)$ are indicated with a circle symbol. Off-axis ICRH cases $\left(R_{I C}-R_{\text {mag }} \sim 0.40 m\right)$ are highlighted. Radiation data ate flux surface averaged and timeaveraged $(\Delta t=1 s)$.

Flat tungsten profile (peaking $~ 1.5$ ) can be achieved at 3-3.5MA with high ICRH power $\left(\mathrm{P}_{\mathrm{ICRH}} \sim 5 \mathrm{MW}\right)$ and high gas rate $\left(\sim 3 \times 10^{22} \mathrm{el} . / \mathrm{s}\right.$ at $3 \mathrm{MA}, \sim 4 \times 10^{22} \mathrm{el} . / \mathrm{s}$ at $\left.3.5 \mathrm{MA}\right)$ at the expense of the global energy confinement (H98y 0.75). More generally, it is found a good correlation between the tungsten core radiation and the normalized confinement time (figure 14). At low plasma current (2.5MA), high $\mathrm{H}$ factor $(\mathrm{H} 98 \mathrm{y}>0.9)$ is achieved as long as the average $\mathrm{W}$ peaking factor is below 2 . This was achieved with a wide range of gas injection rate $\left(1.7-5.7 \times 10^{22} \mathrm{el} . / \mathrm{s}\right)$. It should be noted that in one of these discharges, very strong 
tungsten radiation outside of the $\mathrm{r} / \mathrm{a}=0.5$ surface ('tungsten event') occurs with a weak increase of the core radiation $\left(\sim 0.05 \mathrm{MW} / \mathrm{m}^{3}\right)$ and the core tungsten peaking increases from 1 to only 1.5 . Consistently, the $\mathrm{R} / \mathrm{L}_{\mathrm{ne}}-0.5 \mathrm{R} / \mathrm{L}_{\mathrm{Te}}$ factor is kept negative. At a plasma current exceeding 2.8MA, the tungsten peaking factor always exceed 2 and the $\mathrm{H}$ factor is lower than 0.85 .

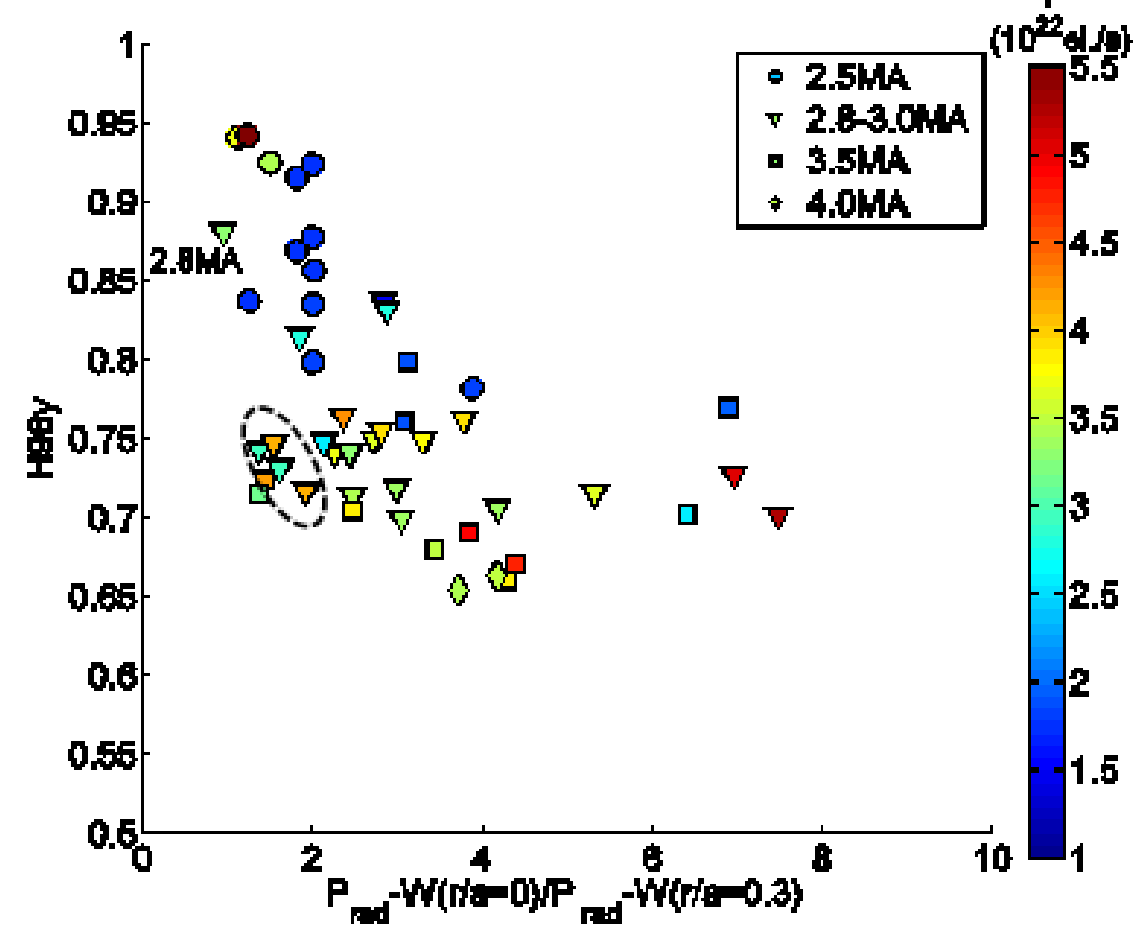

Figure 14. Normalized energy confinement as a function of the tungsten radiation peaking factor for various plasma currents. The 3MA and 3.5MA discharges, highlighted by a circle, have a high helium concentration $\left(n_{\mathrm{He}} \sim 20 \%\right)$ and lower plasma energy due to dilution effect.

\subsection{N2-seeded discharges}

ICRH has been added to discharges $\left(\mathrm{B}_{\mathrm{t}} / \mathrm{I}_{\mathrm{p}}=2.7 \mathrm{~T} / 2.5 \mathrm{MA}\right)$ where nitrogen was used as a radiator aiming at reducing the heat load on the divertor [Giroud 15]. These discharges have been performed with the outer strike point either on the horizontal plate far from the pumping duct $\left(\mathrm{R}_{\mathrm{OSP}}=2.75-2.82 \mathrm{~m}\right)$ or on the vertical plate $\left(\mathrm{R}_{\mathrm{OSP}}=2.88 \mathrm{~m}\right)$ with a higher pumping speed. In the first case, ICRH $\left(\mathrm{P}_{\mathrm{ICRH}}=0-4.5 \mathrm{MW}\right)$ is applied 1 second after the NBI start $\left(\mathrm{P}_{\text {tot }}=18-23 \mathrm{MW}\right)$ whereas in the second case ICRH $\left(\mathrm{P}_{\mathrm{ICRH}}=0-4 \mathrm{MW}\right)$ is delayed with respect of NBI $\left(\mathrm{P}_{\mathrm{tot}}=18-21 \mathrm{MW}\right)$ by 4 seconds. Central IC heating $\left(\mathrm{R}_{\mathrm{IC}}-\mathrm{R}_{\mathrm{mag}} \sim-0.07 \mathrm{~m}\right)$ is applied for all pulses. Deuterium and nitrogen gas rates is in the range of $2.5-7 \times 10^{22} \mathrm{el} . / \mathrm{s}$ and $1.5-4 \times 10^{22} \mathrm{el} . / \mathrm{s}$, respectively. 
For both plasma scenarios, with no ICRH, strong tungsten accumulation $\left(\mathrm{PF}_{0.3} \sim 10\right)$ occurs with strong density peaking $\left(\mathrm{R} / \mathrm{L}_{\mathrm{n}}>2.5\right)$ and finally the central temperature collapses $\left(\mathrm{R} / \mathrm{L}_{\mathrm{Te}}<0\right)$. With ICRH, quasi-stationary discharges are obtained with quite identical normalized density and temperature gradients to those obtained in non-seeded discharges and it results slightly negative values of the $R / L_{n}-0.5 R / L_{T e}$ parameter for $P_{I C R H}$ exceeding 3MW (figure 15-a). Consistently, the tungsten peaking factor $\mathrm{PF}_{0.3}$ decreases from $\sim 7$ (peak value) with no ICRH to $\sim 1.7$ (averaged on two sawtooth periods) with $4.5 \mathrm{MW}$ of RF power (figure 15-b). The main difference between the unseeded and seeded discharges is related to the fraction of power radiated in the main plasma. For the unseeded case, $\mathrm{P}_{\mathrm{rad}}-\mathrm{Bulk} / \mathrm{P}_{\text {tot }}$ is quite low in only NBI-heated discharges (13\%), it exceeds $55 \%$ for the N2-seeded case where most of the radiation is located in the outer half of the plasma (r/a $>0.5)$. With $4.5 \mathrm{MW}$ of ICRH, $\mathrm{P}_{\mathrm{rad}}-\mathrm{Bulk} / \mathrm{P}_{\text {tot }}$ is reduced to $30 \%$ close to the value obtained in the unseeded discharge. This ICRH-heated discharges has an $\mathrm{H}$ factor of 0.83 , slightly larger than for the unseeded case $(\mathrm{H} 98 \mathrm{y}=0.75)$ with quite similar ELM frequency $(50 \mathrm{~Hz}$ vs. $40 \mathrm{~Hz})$.
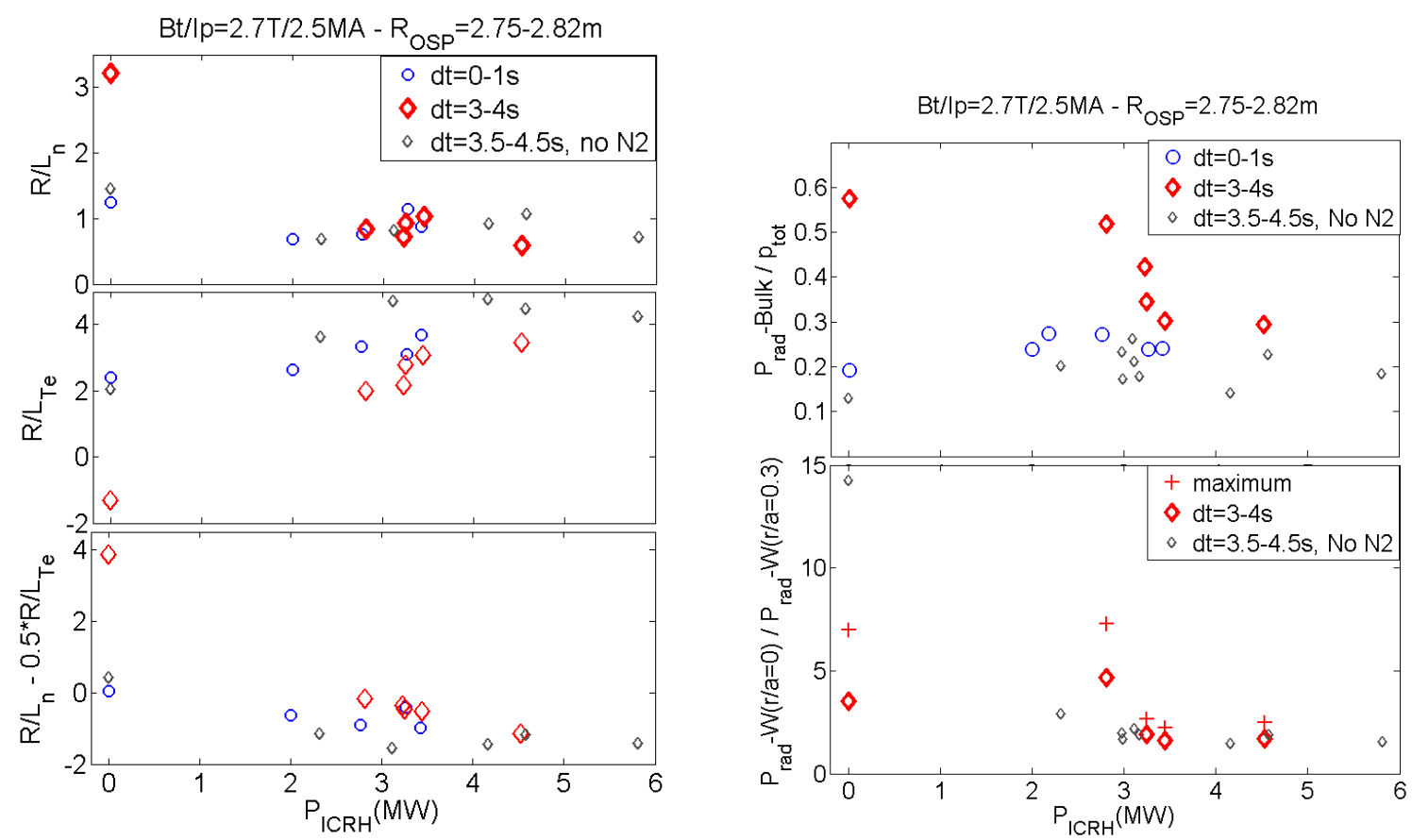

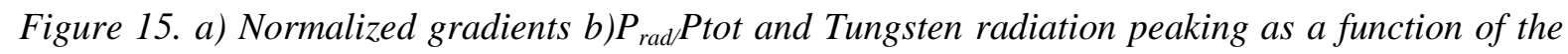
ICRH power. $P_{\text {tot }}=18-23 \mathrm{MW}, F_{t o t}=6-7 \times 10^{22} \mathrm{el} . / \mathrm{s}, F_{N 2}=3-4 \times 10^{22} \mathrm{el} . / \mathrm{s}$, early (circles) and late (large diamonds) after the start of the ICRH power. For comparison, the case of non-seeded discharges $\left(F_{\text {tot }}=1.1-1.6 \times 10^{22}\right.$ el./s) is also shown (small diamonds). 
Similar results are obtained with the outer strike point on the vertical plate, closer to the pumping duct and tungsten peaking, averaged on 2-3 sawtooth periods, is below 2 when the ICRH power is exceeding 3.5MW. In this case with high ICRH power MW), the total (resp. $\mathrm{N} 2$ ) gas rate was varied between 2.7 and $5.4 \times 10^{22} \mathrm{el} . / \mathrm{s}$ (resp. 1.5 and $3.2 \times 10^{22} \mathrm{el} . / \mathrm{s}$ ). The mean tungsten peaking in stationary conditions (2-3s after the start of the ICRH power, 6-7s after the start of the NBI power) decreases from $\sim 2.7$ to $\sim 1.8$ with increasing gas rate (figure 16). At the same time the normalized density gradient $\mathrm{R} / \mathrm{L}_{\mathrm{n}}$ decreases from $\sim 1.2$ to $\sim 0.4$ and $\mathrm{R} / \mathrm{L}_{\mathrm{n}}-0.5 \mathrm{R} / \mathrm{L}_{\mathrm{Te}}$ from $\sim 0$ to $\sim-1.5$. However the $\mathrm{H}$-factor is found to be the highest $\left(\mathrm{H}_{98 \mathrm{y}} \sim 0.9\right)$ for the low gas rate case, thanks to the highest ELM frequency (and lower ELM amplitude) providing a lower impurity source.
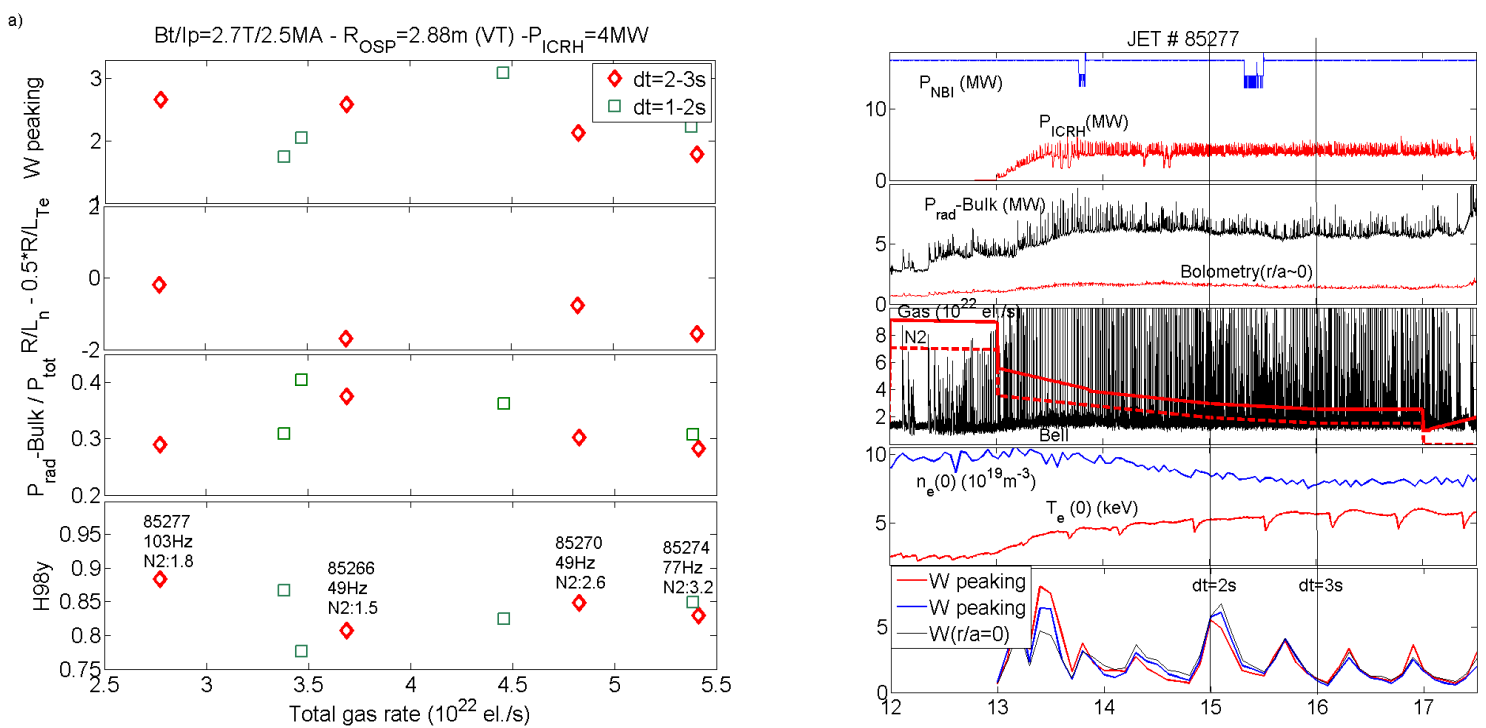

Figure 16. a) Tungsten peaking $P_{\text {rad }} W(r / a=0) / P_{r a d}-W(r / a=0.3), R / L_{n}-0.5 R / L_{T e}$, fraction of radiated power and $H$-factor as a function of the total gas rate $\left(P_{t o t}=20 M W, P_{I C R H}=4 M W\right.$, outer strike point on the vertical target) at 2 time windows. The ELM frequency and the N2 gas rate (in $10^{22}$ el./s) are indicated for the 4 discharges. $b)$ Time traces for the low gas rate case $\left(2.7 \times 10^{22}\right.$ el./s). NBI starts at $t=9$ seconds.

\section{ICRH in JET Hybrid scenario}


Experiments with the hybrid scenario (flat q profile with $\mathrm{q}_{0} \sim 1$ ) were conducted on JET with the ILW at $\mathrm{I}_{\mathrm{p}}=2.5 \mathrm{MA}$ and $\mathrm{B}_{\mathrm{t}}=2.9 \mathrm{~T}$ [Challis 2015]. This scenario allows ICRH in the hydrogen minority heating scheme with $R_{\text {res }}-R_{\text {magn }}=0.08-0.20 \mathrm{~m}$ (LFS), depending on the normalized plasma pressure $\left(\beta_{\mathrm{p}}\right)$. In the reported experiments, the total power is in the 22 $27 \mathrm{MW}$ range, the ICRH power in the $2.8-5.0 \mathrm{MW}$ range. In order to achieve high confinement discharges $(\mathrm{H} 98 \mathrm{y}>1)$, the gas injection rate is reduced $\left(\mathrm{F}=1.0-1.5 \times 10^{22} \mathrm{el} . / \mathrm{s}\right)$. However, with this scenario, the ELM frequency varies in a wide range: for $\mathrm{F}=1.0$ $1.2 \times 10^{22} \mathrm{el} . / \mathrm{s}$, the ELM frequency is low $(<30 \mathrm{~Hz})$ whereas for $\mathrm{F}>1.3 \times 10^{22} \mathrm{el} . / \mathrm{s}$, the ELM frequency generally exceeds $70 \mathrm{~Hz}$ with no evidence of ordering with the total input power. In order to get the requited q-profile, the high power phase is started earlier in the discharge, typically at $\mathrm{t}=5.5 \mathrm{~s}$ for NBI and ICRH when NBI (resp. ICRH) is started at $\mathrm{t}=7 . \mathrm{s}$ (resp.7.5s) in the case of the baseline scenario. As a consequence, the initial density profile is more peaked for discharges in the hybrid scenario case: this normalized density gradient $\mathrm{R} / \mathrm{L}_{\mathrm{n}}$, measured 1s after the start of the high power phase, varies between 2 and 3 when it is in the 1-1.5 range for 3.0-3.5MA baseline discharges in the same range of power (20-26MW).

When the NBI and ICRH powers are applied, the density starts peaking after 2-2.5s the start of this high power phase and tungsten accumulates in the plasma core. The central temperature decreases but the normalized Te gradient is sometime maintained high $\left(\mathrm{R} / \mathrm{L}_{\mathrm{Te}}>4\right)$. The resulting $\mathrm{R} / \mathrm{L}_{\mathrm{n}}-0.5 \mathrm{R} / \mathrm{L}_{\mathrm{Te}}$ parameter is positive (figure $17-\mathrm{a}$ ). No beneficial effect of the ICRH power between 3 and $5 \mathrm{MW}$ on the core radiation $(\mathrm{r} / \mathrm{a}<0.3)$ is observed but the total radiated power in the main plasma decreases strongly with the additional ICRH power: $\mathrm{P}_{\mathrm{rad}}-\mathrm{Bulk} / \mathrm{P}_{\text {tot }}$ decreases from $\sim 70 \%$ to $20 \%$ by just increasing the ICRH power by 2MW (figure 17-b). Tungsten and nickel radiations at mid-radius, derived from the NiXXIII line intensity, decrease identically to the total radiation. 

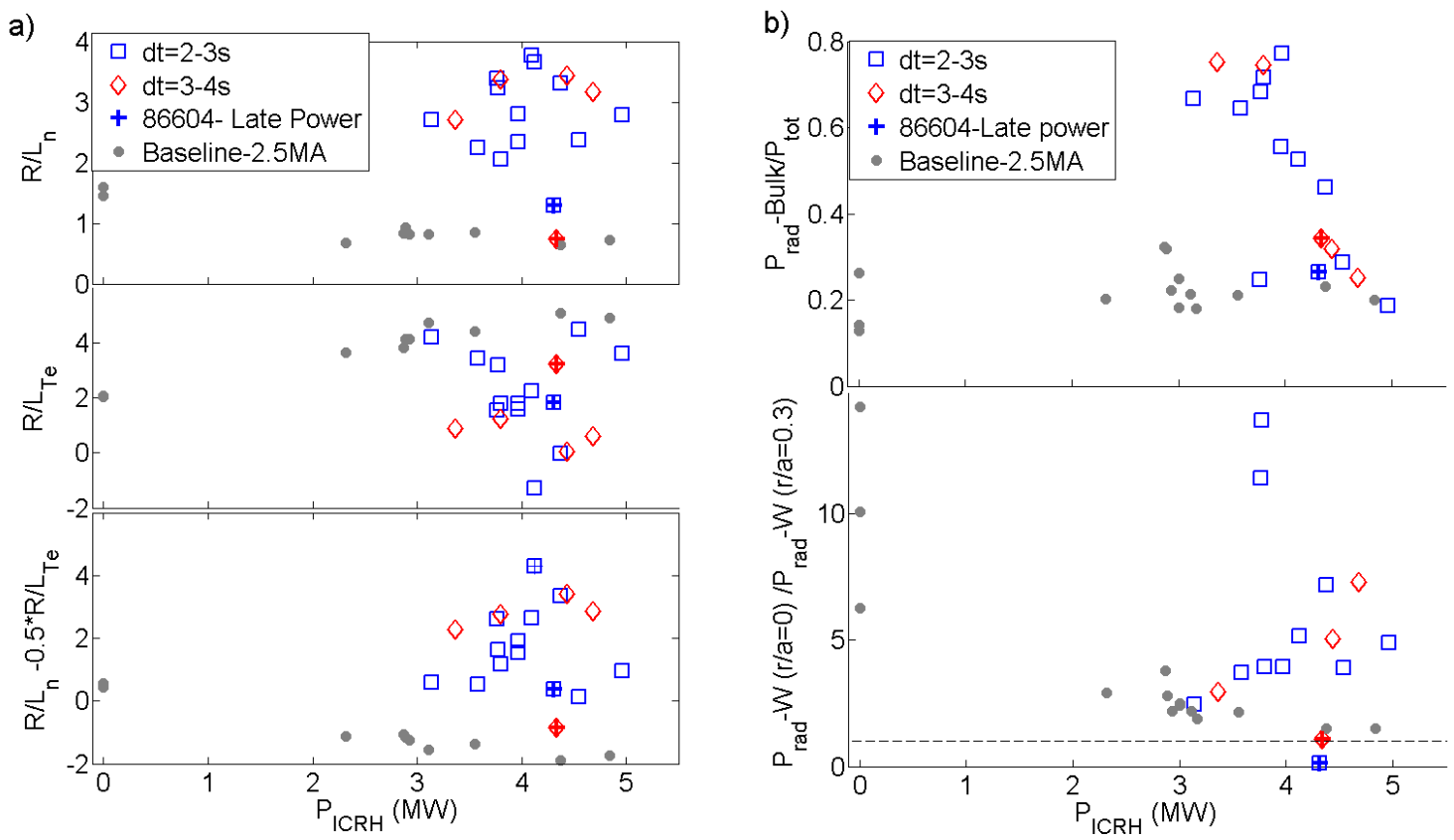

Figure 17) Normalized gradients b) $P_{\text {rad }}$ Bulk/P $P_{\text {tot }}$ and tungsten radiation peaking as a function of the ICRH power for two time slices after the start of the high power phase, $d t=2-3 \mathrm{~s}$ (squares), $d t=3-4 \mathrm{~s}$ (diamonds). $B_{t}=2.9 T, \quad I_{p}=2.5 M A, \quad P_{\text {tot }}=22-27 M W, \quad F_{\text {gas }}=1.0-1.5 \times 10^{22}$ el./s. Baseline discharges (2.7T/2.5MA) are indicated with close circles.

In one case, when the start of the high power phase was delayed by $0.5 \mathrm{~s}\left(\mathrm{t}_{\mathrm{star}}=6 \mathrm{~s}\right)$, the low initial density peaking is maintained and even decreases during the high power phase $\left(R / L_{n}\right.$ decreases from 1.6 to 0.7 ). For this discharge, the tungsten radiation profile is maintained hollow with very low radiation in the core $\left(<0.02 \mathrm{MW} / \mathrm{m}^{3}\right.$ at $\left.\mathrm{r} / \mathrm{a}=0\right)$ for $3 \mathrm{~s}$. This discharge has chirping fishbones and strong 1/1 mode. Conversely, the other discharges have either no MHD activity or a strong 3/2 mode. However, the good confinement of the low tungsten core radiation discharge $\left(\mathrm{H}_{98 \mathrm{y}} \sim 1.1, \beta_{\mathrm{N}} \sim 1.9\right)$ is not sustained beyond $\mathrm{t}-\mathrm{t}_{\text {start }}=3 \mathrm{~s}$ as the current profile evolves and the first sawtooth is triggered at $\mathrm{t}-\mathrm{t}_{\text {start }}=3.5 \mathrm{~s}$.

\section{Discussion and conclusions.}

Ion cyclotron resonance heating in the hydrogen minority scheme in high density plasmas provides heating of both thermal deuterium and electrons with a ratio which does not depart from equipartition by very much after collisional redistribution in a wide range of hydrogen concentration. ICRH modelling also indicates that temperature screening from fast hydrogen ions can be roughly of the same magnitude than the one provided by the thermal deuterium ions, but the uncertainty is large due to the dependency on the derivative of the fast ion 
profile $\mathrm{dT}_{\mathrm{i}} / \mathrm{d} \rho$. The screening is more effective at high hydrogen concentration, although the fast ion temperature decreases with $\mathrm{n}_{\mathrm{H}} / \mathrm{n}_{\mathrm{e}}$.

Tungsten radiation in the plasma core, derived from the soft X-ray diagnostic, has been analyzed on a large data base covering the baseline scenario $\left(\mathrm{I}_{\mathrm{p}}=2.5-4.0 \mathrm{MA}\right)$ and the hybrid scenario $\left(\mathrm{I}_{\mathrm{p}}=2.5 \mathrm{MA}\right)$ with various gas injection rates.

For the baseline scenario, central ICRH is clearly efficient for reducing the tungsten accumulation in the plasma core. Using as a criteria for tungsten accumulation the ratio of $\mathrm{W}$ radiation at $\mathrm{r} / \mathrm{a}=0$ to $\mathrm{r} / \mathrm{a}=0.3$, spatially flux-surface averaged and temporally averaged on a sawtooth period at least 2.5 seconds after the start of the high power phase, flat or modestly peaked tungsten radiation $\left(\mathrm{P}_{\mathrm{rad}}-\mathrm{W}(\mathrm{r} / \mathrm{a}=0) / \mathrm{P}_{\mathrm{rad}}-\mathrm{W}(\mathrm{r} / \mathrm{a}=0.3)<1.8\right)$ profiles are achieved with $\sim 5 \mathrm{MW}$ of RF power either at low plasma current $\left(\mathrm{I}_{\mathrm{p}}=2.5 \mathrm{MA}\right)$ or at higher plasma current $\left(I_{p}=3 M A\right)$ but with higher gas rate. For these H-mode discharges, the global confinement, which is the result of a complex combination of central high $\mathrm{Z}$ impurity content in the core and gas injection acting on the pedestal pressure but also beneficially on the tungsten source by mitigating the ELMs, is the highest $\left(\mathrm{H}_{98, \mathrm{y}} \sim 0.95\right)$ only for the 2.5MA discharges.

In the case of discharges carried out in the frame of the hybrid scenario, with $\mathrm{P}_{\text {ICRH }}$ in the 4$5 \mathrm{MW}$ range, strong tungsten accumulation occurs after $\sim 2$ seconds. This is the correlated with the high initial peaking of the density inherent to this scenario. When this initial density peaking is slightly reduced, very low tungsten concentrations are observed, with hollow profiles maintained for 3 seconds after the start of the high power phase.

Beneficial effect of the fast ion population can be seen from the evolution of tungsten transport and fishbone activity during a sawtooth cycle. This is also illustrated by observation of no (or slow) tungsten accumulation in long sawtooth-free discharge obtained either at low minority concentration (when the energy of the fast ions is the highest) or when the resonance is slightly on the low field side (with possibly optimal effect of the trapped fast ions), but still inside $\mathrm{q}=1$. Fishbone activity is also, and only, observed during the hybrid type discharge with low tungsten concentration.

The tungsten radiation peaking factor is consistent with the parameter $R / L_{n}-0.5 R / L_{T e}$ which is often used as a proxy for neo-classical transport (V/D) of heavy impurities when the poloidal asymmetry arising from toroidal rotation is neglected (and assuming $\mathrm{T}_{\mathrm{e}}=\mathrm{T}_{\mathrm{i}}, \mathrm{n}_{\mathrm{e}}=\mathrm{n}_{\mathrm{i}}$ ). The amplitude of this parameter is consistent with the $\mathrm{W}$ peaking factor for the various scenarios. In the baseline scenario, when ICRH is applied from the start of the high power 
phase, density peaking does not evolve by much when the ICRH power is increased from $3 \mathrm{MW}$ to $6 \mathrm{MW}$ and the improvement is caused by the $\mathrm{R} / \mathrm{T}_{\mathrm{e}}$ factor. In the hybrid scenario, the initial normalized density gradient $\mathrm{R} / \mathrm{L}_{\mathrm{Te}}$, before the high power phase, is typically 2-3 times larger than in the baseline scenario case and ICRH power, up to $5 \mathrm{MW}$, is not efficient to reverse the sign of the neo-classical transport parameter $\left(R / L_{n}-0.5 R / L_{T e}>0\right)$. It is striking that by changing slightly the timing of the high power phase which decreases the initial $R / L_{n}$ by only $\sim 10 \%$ (with ICRH power increased by $\sim 10 \%$ ), the density peaking further decreases steadily during the discharge to lower values than obtained for most of the pulses in the baseline scenario $\left(\mathrm{R} / \mathrm{L}_{\mathrm{n}} \sim 0.7\right)$.

JET is preparing the D-T pulses which will be performed at higher power $\left(\mathrm{P}_{\text {tot }}>35 \mathrm{MW}\right)$. With the addition of the ILA antenna [Durodie 12] and further optimization of the ICRH system, $8-9 \mathrm{MW}$ could be achievable with a gas rate of $\sim 1 \times 10^{20} \mathrm{el} . / \mathrm{s}$. We can expect further improvement for controlling the tungsten concentration in the plasma core for the baseline scenario. The effect of ICRH for the hybrid scenario is more speculative, but sensitivity of the density peaking to the details of the scenario could be an opportunity for the effective control of tungsten accumulation while keeping good energy confinement for 5 seconds.

\section{Acknowledgements}

This work has been carried out within the framework of the EUROfusion Consortium and has received funding from the EURATOM research and training programme 2014-2018 under grant agreement No 633053. The views and opinions expressed herein do not necessarily reflect those of the European Commission.

\section{References}

[Angioni 2014] Nucl. Fusion 54 (2014) 083028 (26pp) http://iopscience.iop.org/07413335/57/1/014031/pdf/0741-3335_57_1_014031.pdf

[Angioni 2015] Physics of Plasmas 22, 055902 (2015)

http://scitation.aip.org/content/aip/journal/pop/22/5/10.1063/1.4919036 
[Bilato 2014] R. Bilato, O. Maj and C. Angioni, Nucl. Fusion 54 (2014) 072003 (4pp) http://iopscience.iop.org/article/10.1088/0029-5515/54/7/072003/pdf

[Bobkov 2013] V. Bobkov et al., Journal of Nucl. Materials 438 (2013) S160-S165, http://www.sciencedirect.com/science/article/pii/S0022311513000524

[Breizman 2011] B N Breizman and S E Sharapov, Plasma Phys. Control. Fusion 53 (2011) 054001 (39pp) http://iopscience.iop.org/article/10.1088/0741-3335/53/5/054001/pdf

[Casson 2015] Plasma Phys. Control. Fusion $57 \quad$ (2015) 014031 (10pp) http://iopscience.iop.org/0741-3335/57/1/014031/pdf/0741-3335_57_1_014031.pdf

[Challis 2015] Challis C. et al., Nucl. Fusion 55 (2015) 053031 (18pp), http://iopscience.iop.org/article/10.1088/0029-5515/55/5/053031/pdf

[Czarnecka 2014 ] A. Czarnecka et al., AIP Conf. Proc. 1580 (2014) p.227

[Dumont 2009] Dumont R.J. 2009 Nucl. Fusion 49075033

http://iopscience.iop.org/article/10.1088/0029-5515/49/7/075033/pdf

[Dumont 2013] R.J. Dumont and D. Zarzoso 2013 Nucl. Fusion 53013002 http://iopscience.iop.org/article/10.1088/0029-5515/53/1/013002/pdf

[Durodié 12] F.Durodié et al., Plasma Phys. Control. Fusion 54 (2012) 074012 (16pp) http://iopscience.iop.org/article/10.1088/0741-3335/54/7/074012/pdf

[Giroud 2015] Giroud C. et al., Plasma Phys. Control. Fusion 57 (2015) 035004 (20pp) http://iopscience.iop.org/0741-3335/57/3/035004/pdf/0741-3335_57_3_035004.pdf

[Goniche 2014] Goniche M et al, Proc. 41st EPS Conf. Plasma Physics, Berlin, 23-27 June 2014, O4.129, http://ocs.ciemat.es/EPS2014ABS/pdf/O4.129.pdf 
[Graves 2015] J.P.Graves et al., Plasma Phys. Control. Fusion 57 (2015) 014033 (14pp) http://iopscience.iop.org/article/10.1088/0741-3335/57/1/014033/pdf

[Jacquet 14] P. Jacquet et al., Physics of Plasmas 21 (2014) pp.061510, http://scitation.aip.org/content/aip/journal/pop/21/6/10.1063/1.4884354

[Lerche 14] Lerche E. et al., Proc. 25th Int. Conf. on Fusion Energy (Saint Petersburg, Russia, 2014) EX/P5-22, submitted to Nuclear Fusion

[Lerche 14b] Lerche E. et al., Journal of Nucl. Materials 463 (2015) 634-639 http://www.sciencedirect.com/science/article/pii/S0022311514007582

[Klepper 13], C. Klepper et al., Journal of Nucl. Materials 438 (2013) S594-S598, http://www.sciencedirect.com/science/article/pii/S0022311513001323

[Lennholm 15] M. Lennholm et al., Nucl. Fusion 56 (2016) 016008

http://iopscience.iop.org/article/10.1088/0029-5515/56/1/016008/pdf

[Mantica 2014] P.Mantica, E.Puiatti, M.L.Reinke, M.Romanelli and JET EFDA contributors, Proc. $41^{\text {th }}$ European Physical Society Conference on Plasma Physics, Berlin 2014, P1.107 (2014), http://ocs.ciemat.es/EPS2014PAP/pdf/P1.017.pdf.

[Mantica 2015] P. Mantica F.J.Casson, M.Valisa, C.Angioni, R.Bilato, E.Belonohy, C.Giroud, M.Goniche ,N.Hawkes, E. Lerche and JET contributors, Proc. 42th European Physical Society Conference on Plasma Physics, Lisbon 2015, P1.101 (2015), http://ocs.ciemat.es/EPS2015PAP/pdf/P1.101.pdf.

[Mantsinen 1999] Mantsinen M. et al , Plasma Phys. Control. Fusion 41 (1999) 843-865. http://iopscience.iop.org/article/10.1088/0741-3335/41/7/301/pdf 
[Mantsinen 2015] Mantsinen M. et al ; Proc. 42th European Physical Society Conference on Plasma Physics, Lisbon 2015, P2.171 (2015),

http://ocs.ciemat.es/EPS2015PAP/pdf/P2.171.pdf

[Nave 2003], M.F.F. Nave et al., Nucl. Fusion 43 (2003) 1204-1213 http://iopscience.iop.org/article/10.1088/0029-5515/43/10/023/pdf

[Nunes 2014], I.Nunes et al., Plasma Physics and Control. Fusion 58 (2016) 014034 (10 pp.) http://iopscience.iop.org/article/10.1088/0741-3335/58/1/014034/pdf

[Pütterich 2012] T. Pütterich, tungsten control in JET," in IAEA Fusion Energy Conference, San Diego, EX/P3-15 (2012), http://wwwnaweb.iaea.org/napc/physics/FEC/FEC2012/papers/193_EXP315.pdf.

[Pütterich 2013] T.Pütterich et al, Plasma Phys. Control. Fusion 55 (2013) 124036, http://iopscience.iop.org/0741-3335/55/12/124036/pdf/0741-3335_55_12_124036.pdf

[Valisa 2011] M.Valisa et al. ,Nucl. Fusion $51 \quad$ (2011) 033002 (11pp) http://m.iopscience.iop.org/0029-5515/51/3/033002/pdf/0029-5515_51_3_033002.pdf

[Van Eester 14] D. Van Eester et al., EPS Confernce, Proc. $41^{\text {st }}$ EPS Conf. Plasma Physics, Berlin, June 2014, http://ocs.ciemat.es/EPS2014ABS/pdf/P1.002.pdf 